ANNALES

POLONICI MATHEMATICI

$90.2(2007)$

\title{
A Littlewood-Paley type inequality with applications to the elliptic Dirichlet problem
}

\author{
by Caroline Sweezy (Las Cruces, NM)
}

\begin{abstract}
Let $L$ be a strictly elliptic second order operator on a bounded domain $\Omega \subset \mathbb{R}^{n}$. Let $u$ be a solution to $L u=\operatorname{div} \vec{f}$ in $\Omega, u=0$ on $\partial \Omega$. Sufficient conditions on two measures, $\mu$ and $\nu$ defined on $\Omega$, are established which imply that the $L^{q}(\Omega, d \mu)$ norm of $|\nabla u|$ is dominated by the $L^{p}(\Omega, d v)$ norms of $\operatorname{div} \vec{f}$ and $|\vec{f}|$. If we replace $|\nabla u|$ by a local Hölder norm of $u$, the conditions on $\mu$ and $\nu$ can be significantly weaker.
\end{abstract}

Introduction. The intent of this paper is to establish sufficient conditions on two measures, $\mu$ and $\nu$, defined on a bounded domain $\Omega$ in $\mathbb{R}^{n}$, $n \geq 3$, so that

$$
\left(\int_{\Omega}|\nabla u(x)|^{q} d \mu(x)\right)^{1 / q} \leq C\left(\int_{\Omega}\left(|\operatorname{div} \vec{f}(x)|^{p}+|\vec{f}(x)|^{p}\right) d \nu(x)\right)^{1 / p}
$$

if $2<p \leq q<2+\varepsilon$, for any function $u(x)$ that is a weak solution to

$$
\left\{\begin{array}{l}
L u(x)=\operatorname{div} \vec{f}(x), \quad x \in \Omega, \\
\left.u\left(x^{\prime}\right)\right|_{\partial \Omega}=0 .
\end{array}\right.
$$

Here $\Omega$ is assumed to satisfy an exterior cone condition, $\vec{f} \in H^{1}(\Omega)$, and

$$
L=\sum_{1 \leq i, j \leq n} \frac{\partial}{\partial x_{i}}\left(a_{i, j}(x) \frac{\partial}{\partial x_{j}}\right)
$$

2000 Mathematics Subject Classification: 35J25, 42B25.

Key words and phrases: elliptic equations, Lipschitz domains, Littlewood-Paley type inequalities.

Funding was provided by the National Science Foundation-funded ADVANCE Institutional Transformation Program at New Mexico State University, fund \#NSF0123690. Any opinions, findings and conclusions or recommendations expressed in this material are those of the author and do not necessarily reflect the views of the National Science Foundation. 
is a strictly elliptic divergence form operator, in other words,

$$
\sum_{1 \leq i, j \leq n} \xi_{i} a_{i, j}(x) \xi_{j} \geq \lambda|\xi|^{2} \quad \text { for some } \lambda>0 \text { and all } x \in \Omega .
$$

$L$ 's coefficients are assumed to be symmetric, $a_{i, j}(x)=a_{j, i}(x)$, and bounded and measurable on $\Omega$. Since $\Omega$ is bounded, for $\mu(\Omega)<\infty$ and $\nu(\Omega)<\infty$ (this is implied by the conditions that will be imposed on $\mu$ and $\nu$ ), we can extend (1) to be valid for indices $0<q<2+\varepsilon$ and $2<p<\infty$, simply by using Hölder's inequality. The constant $C$ in (1) depends on $\Omega$ in any case, so this does not restrict the result too much for the wider range of indices. However, the presence of the gradient of $u$ does introduce a restriction. Following a suggestion of Professor Wheeden, we replace $|\nabla u(x)|$ by a local Hölder norm of $u$ at $x$, and prove sufficient conditions on $\mu$ and $\nu$ so that for $0<q<\infty, 1<p<\infty$,

$$
\left(\int_{\Omega}\|u(x)\|_{H^{\alpha}}^{q} d \mu(x)\right)^{1 / q} \leq C\left(\int_{\Omega}\left(|\operatorname{div} \vec{f}(x)|^{p}\right) d \nu(x)\right)^{1 / p},
$$

with

$$
\|u(x)\|_{H^{\alpha}}=\sup _{y \in P(x)} \frac{|u(x)-u(y)|}{|x-y|^{\alpha}}
$$

where

$$
\begin{aligned}
P(x) & =\left\{z \in \Omega:\left|x_{i}-z_{i}\right| \leq b \delta(x), i=1, \ldots, n\right\}, \\
\delta(x) & =\operatorname{dist}(x, \partial \Omega) .
\end{aligned}
$$

The constant $b<1$ is fixed and chosen so that both $P(x)$ and $\eta P(x)=$ $\left\{z \in \Omega:\left|x_{i}-z_{i}\right| \leq \eta b \delta(x), i=1, \ldots, n\right\}$ for $\eta_{0} \geq \eta>1, \eta_{0}>4$ fixed, are Whitney-type cubes in $\Omega$ centered at $x$. Corresponding results for solutions to the homogeneous Dirichlet problem

$$
\left\{\begin{array}{l}
L u(x)=0, \quad x \in \Omega \\
\left.u\left(x^{\prime}\right)\right|_{\partial \Omega}=f\left(x^{\prime}\right)
\end{array}\right.
$$

are proved in $[\mathrm{S}]$.

Combining the results of this paper with those in $[\mathrm{S}]$ and $[\mathrm{SW}]$, and using superposition, we have established sufficient conditions on measures $\mu$ and $\nu$ on $\Omega$, and on a boundary measure, $\varrho d \omega$, so that for $\|u(x)\|$, which denotes either $|\nabla u(x)|$ or $\|u(x)\|_{H^{\alpha}}$, it follows that

$$
\begin{aligned}
& \left(\int_{\Omega}\|u(x)\|^{q} d \mu(x)\right)^{1 / q} \\
\leq & C\left(\left(\int_{\Omega}\left(|\operatorname{div} \vec{f}(x)|^{p}+|\vec{f}(x)|^{p}\right) d \nu(x)\right)^{1 / p}+\left(\int_{\partial \Omega}\left|g\left(x^{\prime}\right)\right|^{r} \varrho\left(x^{\prime}\right) d \omega\left(x^{\prime}\right)\right)^{1 / r}\right)
\end{aligned}
$$


for solutions to

$$
\left\{\begin{array}{l}
L u(x)=\operatorname{div} \vec{f}(x), \quad x \in \Omega, \\
\left.u\left(x^{\prime}\right)\right|_{\partial \Omega}=g\left(x^{\prime}\right),
\end{array}\right.
$$

if $\Omega$ is a bounded Lipschitz domain in $\mathbb{R}^{n}, n \geq 3$. $\omega=\omega^{x_{0}}$ is the elliptic measure on $\partial \Omega$ generated by $L$, measured from a fixed point $x_{0}$ interior to $\Omega$. So for $L=\Delta, \omega$ is harmonic measure.

The method of obtaining the conditions on the measures follows that of Wheeden and Wilson [WW] in using dual operator norms. This argument utilizes a norm inequality that derives from Littlewood-Paley theory. The crucial Littlewood-Paley type inequality is proved in Theorem 1 for functions of the form

$$
h(x)=\sum_{J \in \mathcal{F}} \lambda_{J} \varphi_{(J)}(x), \quad \lambda_{J} \in \mathbb{R},
$$

using methods closely allied to those in [W], [SW]. The $\varphi_{(J)}(x)$ are members of a family of functions that have certain decay and cancellation properties, and $\mathcal{F}$ is a finite family of dyadic cubes. The details about the $\varphi_{(J)}(x)$ are stated below in $(\mathrm{a}),\left(\mathrm{a}^{\prime}\right),(\mathrm{b})$ and $(\mathrm{c})$. To prove the $\varphi_{(J)}(x)$ satisfy the necessary conditions, we utilize geometric properties of elliptic Green's functions on rough domains, proved by Grüter and Widman [GW] (see also $[\mathrm{K}]$ ).

The results presented in this paper stem from a considerable body of work. References for situations in which an inequality of the form

$$
\left(\int_{\Omega}|\nabla u(x)|^{q} d \mu(x)\right)^{1 / q} \leq\left(\int_{\partial \Omega}\left|g\left(x^{\prime}\right)\right|^{p} v\left(x^{\prime}\right) d \omega\left(x^{\prime}\right)\right)^{1 / p}
$$

holds when $u$ is harmonic are given in [SW]. The history for semi-discrete Littlewood-Paley results is mentioned in [W]. Wheeden and Wilson dealt with the case of the Dirichlet problem in the upper half space for harmonic $u(x)$. Sweezy and Wilson later found sufficient conditions for $\mu$ and $\varrho d \omega$ to ensure (4) for harmonic gradients on Lipschitz domains; they found that similar techniques allowed them to deal with elliptic functions on rough domains. A key part of their argument consisted in establishing a LittlewoodPaley type inequality for functions of the form $\sum_{J \in \mathcal{F}} \lambda_{J} \varphi_{(J)}(x)$ with minimal smoothness conditions assumed for the $\varphi_{(J)}$ and for the domain. They accomplished this by an argument in the spirit of Wilson's method of proving a semi-discrete Littlewood-Paley type inequality on $\mathbb{R}^{n}$ for smoother functions (see $[\mathrm{W}]$ ). The fact that the operator $L$ has an associated kernel function which has geometric decay was an essential ingredient to their proof. It remains an important ingredient in the case of a solution to the inhomogeneous equation.

In Section 1 of this paper the two main theorems are stated. To avoid becoming immersed in technical details too early we leave the proof of The- 
orem 1 until the last section of the paper. Section 2 contains the proof of Theorem 2, assuming that Theorem 1 is valid. Theorem 3 for a local Hölder norm of a solution $u$ instead of the gradient of $u$ is stated and proved after Theorem 2 in that section. Section 3 presents the proof of Theorem 1, the Littlewood-Paley type inequality.

1. The main results. To state the first main theorem, the LittlewoodPaley type inequality, we need to establish some definitions. First recall that a measure $\sigma$ defined on a domain $D$ is said to be $A^{\infty}$ with respect to Lebesgue measure if for any cube $Q \subset D$ and any measurable subset $E$ of $Q$, there are fixed constants $C_{0}$ and $\kappa>0$ so that

$$
\left(\frac{\sigma(E)}{\sigma(Q)}\right)^{\kappa} \leq C_{0} \frac{|E|}{|Q|} \quad(\text { see }[\mathrm{CF}])
$$

We will utilize a family $\mathfrak{D}$ of dyadic cubes which includes all dyadic subcubes of a given (large) cube $Q_{0}$. When $Q$ is a cube, $l(Q)$ will denote the side length of $Q$. If $Q$ is a region that is comparable to a cube, say, $Q$ is the image of an actual Euclidean cube under a Lipschitz map, then $l(Q)$ denotes a length comparable to the side length of the pre-image cube. One could also take $l(Q)$ to be the diameter of any such region. $Q_{0}$ is chosen so that $\Omega \subset Q_{0}$ and $l\left(Q_{0}\right) \sim \operatorname{diam}(\Omega)$. In order to have $\bigcup_{I \in \mathfrak{D}} I$ cover $\Omega$ completely, we take the dyadic subcubes $I$ to be half closed, i.e. of the form $\left[a_{1}, b_{1}\right) \times \cdots \times\left[a_{n}, b_{n}\right) . \mathcal{W}$ is a collection of special dyadic cubes from $\mathfrak{D} ;$ these are Whitney-type cubes that lie inside $\Omega$, are pairwise disjoint, and cover the interior of $\Omega . I \in \mathcal{W}$ implies that $l(I) \simeq \operatorname{dist}(I, \partial \Omega)$, but the $I \in \mathcal{W}$ may be subcubes of a fixed proportion to the usual Whitney cube decomposition of $\Omega$. We need to be sure that $\beta I$ (the $\beta$-dilate of $I$, that is, the cube concentric with $I$ and of side length $\beta l(I))$ is also a Whitney cube for any $1 \leq \beta<\eta_{0}, \eta_{0}>4$. The point $x_{J}$ will denote the geometric center of the dyadic cube $J$, and $\delta(x)=\operatorname{dist}(x, \partial \Omega)$. by

When $f(x)=\sum_{J \in \mathcal{F}} \lambda_{J} \varphi_{(J)}(x)$, the function $g^{*}(f)(x)=g^{*}(x)$ is defined

$$
g^{*}(x)=\left(\sum_{J \in \mathcal{F}} \frac{\lambda_{J}^{2}}{|J|}\left(1+\frac{\left|x-x_{J}\right|}{l(J)}\right)^{-n}\right)^{1 / 2} .
$$

It is a discrete version of the $g_{\lambda}^{*}$ function of classical Littlewood-Paley theory. Notice that the order of decay in $g^{*}$, as defined here, is slightly less than that said to be optimal in [W]. The reason for this is the order of decay for the Green function, $G(x, y)$, which appears in $u(x)=\int_{\Omega} G(x, y) \operatorname{div} \vec{f}(y) d y$, the integral representation for solutions to Poisson's equation. 
The four conditions that will be assumed to hold for the family $\left\{\varphi_{(J)}(x)\right\}$ are:

(a) $\left|\varphi_{(J)}(x)\right| \leq C l(J)^{2-n / 2}\left(1+\frac{\left|x-x_{J}\right|}{l(J)}\right)^{2-n} \quad$ for all $x \in \Omega$,

(a' $^{\prime} \quad\left|\varphi_{(J)}(x)\right| \leq C \delta(x)^{\alpha} l(J)^{2-n / 2-\alpha}\left(1+\frac{\left|x-x_{J}\right|}{l(J)}\right)^{2-n-\alpha} \quad$ for all $x \in \Omega$,

$$
\begin{aligned}
\left|\varphi_{(J)}(x)-\varphi_{J}(y)\right| \leq C|x-y|^{\alpha} l(J)^{2-n / 2-\alpha} & \\
\times & \left(1+\frac{\left|x-x_{J}\right|}{l(J)}+\frac{\left|y-x_{J}\right|}{l(J)}\right)^{2-n-\alpha} \quad \text { for all } x, y \in \Omega, \\
& \int\left|\sum_{J \in \mathcal{F}} \lambda_{J} \varphi_{(J)}(x)\right|^{2} d x \leq C \sum_{J \in \mathcal{F}} \lambda_{J}^{2} .
\end{aligned}
$$

THeOREM 1. Suppose that $f(x)=\sum_{J \in \mathcal{F}} \lambda_{J} \varphi_{(J)}(x)$ is a function defined on $\Omega$, where $\mathcal{F}$ is a finite set of dyadic cubes from $\mathcal{W}$, and the $\left\{\varphi_{(J)}\right\}_{J \in \mathcal{F}}$ are a family of functions that satisfy conditions (a), ( $\left.\mathrm{a}^{\prime}\right),(\mathrm{b})$, and (c), and $\varphi_{(J)}(x)=0$ if $x \in Q_{0} \backslash \Omega$. Then, if $\sigma \in A^{\infty}\left(Q_{0}, d x\right)$, there is a constant $C=C\left(n, \alpha, p, \Omega, \kappa, C_{0}\right)$ such that, for any $0<p<\infty$,

$$
\|f\|_{L^{p}\left(Q_{0}, d \sigma\right)} \leq C\left\|g^{*}\right\|_{L^{p}\left(Q_{0}, d \sigma\right)} .
$$

The major application of Theorem 1 of concern here is to demonstrate sufficient conditions on measures $\mu$ and $\nu$ so that (1) is valid. To state these conditions we need to recall what it means for a measure to satisfy a reverse Hölder condition on a domain $D$ with respect to Lebesgue measure. This is written as $\mu \in B^{r}(D, d x)$, with $r>1$, if for every cube $Q \subset D$,

$$
\left(\frac{1}{|Q|} \int_{Q}\left(\frac{d \mu}{d x}\right)^{r} d x\right)^{1 / r} \leq C\left(\frac{1}{|Q|} \int_{Q} \frac{d \mu}{d x} d x\right) .
$$

It is true that any measure satisfying a reverse Hölder condition with respect to Lebesgue measure is also an $A^{p}$ measure on $D$, for some exponent $p$. (A non-negative $L_{\text {loc }}^{1}(D)$ function $w$ is in $A^{p}(D, d x)$ if

$$
\left(\frac{1}{|Q|} \int_{Q} w d x\right)\left(\frac{1}{|Q|} \int_{Q}\left(\frac{1}{w}\right)^{p^{\prime}-1} d x\right)^{1 /\left(p^{\prime}-1\right)} \leq A_{0}
$$

for all cubes $Q \subset D$.) A measure $\mu$ is in $A^{p}(D, d x)$ if $d \mu / d x \in A^{p}(D, d x)$.) We let $p^{\prime}$ denote the Hölder conjugate index for $p$, that is, $1 / p+1 / p^{\prime}=1$. Any measure that is either an $A^{p}$ measure on $D$, or satisfies a reverse Hölder condition on $D$, is also an $A^{\infty}$ measure on $D$ (see $[\mathrm{CF}]$ ). 
To avoid cluttering up the statement of Theorem 2 we define, for any dyadic cube $Q_{j}$ in $\mathfrak{D}$,

$$
\begin{aligned}
M\left(Q_{j}\right)=\max \left\{l\left(Q_{j}\right)^{n / 2+1}\right. & \left(\int_{2 Q_{j}}\left(\frac{d \nu}{d x}\right)^{-2 /(p-2)} d x\right)^{(p-2) / 2 p}, \\
& \left.\left(\int_{Q_{0}}\left(1+\frac{\left|y-x_{Q_{j}}\right|}{l\left(Q_{j}\right)}\right)^{-n p^{\prime} / 2} d \sigma(y)\right)^{1 / p^{\prime}}\right\} .
\end{aligned}
$$

Suppose that $L=\sum_{1 \leq i, j \leq n}\left(\partial / \partial x_{i}\right)\left(a_{i, j}(x) \partial / \partial x_{j}\right)$ is a strictly elliptic divergence form operator on the domain $\Omega$, as described above. Suppose also that $\mu$, a Borel measure defined on $\Omega$, satisfies a reverse Hölder condition of order $((q+\varepsilon) / q)^{\prime}$ with respect to Lebesgue measure on $\Omega$, i.e. $\mu \in B^{(q+\varepsilon) / \varepsilon}(\Omega, d x)$.

Theorem 2. Let $\mu$ and $\nu$ be Borel measures on $\Omega$ with $\nu$ finite and absolutely continuous with respect to Lebesgue measure, and suppose that $d \sigma(x)=(d \nu / d x)^{1-p^{\prime}} d x$ satisfies the condition $A^{\infty}(\Omega, d x)$. Let $u$ be a solution to $L u=\operatorname{div} \vec{f}$ on $\Omega,\left.u\right|_{\partial \Omega}=0, \vec{f} \in H^{1}(\Omega)$. If there is a constant $C_{0}>0$ so that for every dyadic cube $Q_{j}$ in $\mathcal{W}$,

$$
\mu\left(Q_{j}\right)^{1 / q} M\left(Q_{j}\right) \leq C_{0} l\left(Q_{j}\right)^{n+1},
$$

then there is a constant $C=C\left(n, p, q, \alpha, b, \kappa, \Omega, \lambda, \eta_{0}, \varepsilon\right)$ such that

$$
\left(\int_{\Omega}|\nabla u(x)|^{q} d \mu(x)\right)^{1 / q} \leq C\left(\int_{\Omega}\left(|\operatorname{div} \vec{f}(x)|^{p}+|\vec{f}(x)|^{p}\right) d \nu(x)\right)^{1 / p}
$$

for $2<p \leq q<2+\varepsilon$. For $0<q \leq q_{0}$ and $2<p_{0} \leq p<\infty$, the same inequality is valid upon replacing $C$ by $C \mu(\Omega)^{1 / q-1 / q_{0}} \nu(\Omega)^{1 / p_{0}-1 / p}$, $C=C\left(p_{0}, q_{0}, n, \alpha, b, \kappa, \Omega, \lambda, \eta_{0}, \varepsilon\right)$, for some fixed pair of indices $2<p_{0} \leq$ $q_{0}<2+\varepsilon$.

We will start with a brief discussion of the condition on the measures $\mu$ and $\nu$ given in Theorem 2. Then we will prove Theorem 2 assuming that Theorem 1 is valid. Subsequently we prove Theorem 3, the version of Theorem 2 with $\|u(x)\|_{H^{\alpha}}$ replacing $|\nabla u(x)|$ in (1). The companion result, sufficient conditions on a measure $\mu$ on $\Omega$ and a boundary measure $\varrho d \omega$ on $\partial \Omega$ so that \|\|$u(x)\left\|_{H^{\alpha}}\right\|_{L^{q}(\Omega, d \mu)} \leq C\|g\|_{L^{p}(\partial \Omega, \varrho d \omega)}$ when $L u=0$ in $\Omega,\left.u\right|_{\partial \Omega}=g$, is proved in $[\mathrm{S}]$.

2. Proof of Theorem 2. The condition on the measures $\mu$ and $\nu$ given in Theorem 2 may look complicated, but in fact it is closely related to well known properties of measures such as $A^{p}$ conditions, geometric decay and the concept of Carleson measures. To gain an idea of what the condition in Theorem 2 can mean for the relation between $\mu$ and $\nu$, we look at some 
simple examples. The domain $\Omega$ is bounded, so we need only consider the case when $l\left(Q_{j}\right) \leq 1$.

If $\nu$ is taken to be Lebesgue measure, then Theorem 2's condition becomes that $\mu$, on the Whitney-type cubes $Q_{j}$ in $\Omega$, must satisfy $\mu\left(Q_{j}\right)^{1 / q} \leq$ $C l\left(Q_{j}\right)^{n / 2}$ with $C=C\left(p^{\prime}, n, \operatorname{diam}(\Omega)\right)$. This follows from taking

$$
M\left(Q_{j}\right)=\left(\int_{Q_{0}}\left(1+\frac{\left|y-x_{Q_{j}}\right|}{l\left(Q_{j}\right)}\right)^{-n p^{\prime} / 2} d \sigma(y)\right)^{1 / p^{\prime}}
$$

and performing a standard estimate of the integral over $Q_{0}$ by dividing $Q_{0}$ into dyadic annular regions centered at $Q_{j}$. An elementary calculation shows that this condition also implies that $\mu\left(Q_{j}\right)^{1 / q}$ multiplied by the other term,

$$
l\left(Q_{j}\right)^{n / 2+1}\left(\int_{2 Q_{j}}(d \nu / d x)^{-2 /(p-2)} d x\right)^{(p-2) / 2 p},
$$

in the definition of $M\left(Q_{j}\right)$, is also less than or equal to $C l\left(Q_{j}\right)^{n+1}$ since $d \nu / d x=1$.

As a second example, consider letting $p=q=4$, and take $\mu=\nu$. The requirement that

$$
\mu\left(Q_{j}\right)^{1 / q} \cdot l\left(Q_{j}\right)^{n / 2+1}\left(\int_{Q_{j}}(d \nu / d x)^{-2 /(p-2)} d x\right)^{(p-2) / 2 p}
$$

be bounded by $C l\left(Q_{j}\right)^{n+1}$ turns out to be equivalent to the following $A^{2}$-type condition:

$$
\frac{1}{\left|Q_{j}\right|} \int_{Q_{j}}(d \mu / d x) d x \cdot\left(\frac{1}{\left|Q_{j}\right|} \int_{2 Q_{j}}(d \mu / d x)^{-1} d x\right) \leq C .
$$

(In fact we know that $\mu$ is an $A^{2}$ measure by the reverse Hölder condition on $\mu$.) The second condition, that

$$
\mu\left(Q_{j}\right)^{1 / q} \cdot\left(\int_{Q_{0}}\left(1+\left|y-x_{Q_{j}}\right| / l\left(Q_{j}\right)\right)^{-n p^{\prime} / 2} d \sigma(y)\right)^{1 / p^{\prime}} \leq C l\left(Q_{j}\right)^{n+1},
$$

does not have an exact interpretation as a well known measure condition, but it too can be viewed as a weighted version of an $A^{p}$-type condition with vanishing trace.

To prove Theorem 2 we start by dividing the integral $\|\nabla u\|_{L^{q}(\Omega, d \mu)}^{q}$ into a sum of integrals over Whitney cubes from $\mathcal{W}$ : 


$$
\begin{aligned}
& \int_{\Omega}|\nabla u(x)|^{q} d \mu(x)=\sum_{Q_{j} \in \mathcal{W}} \int_{Q_{j}}|\nabla u(x)|^{q} d \mu(x) \\
& \quad \leq \sum_{Q_{j} \in \mathcal{W}}\left(\int_{Q_{j}}|\nabla u(x)|^{q+\varepsilon} d x\right)^{q /(q+\varepsilon)}\left(\int_{Q_{j}}\left(\frac{d \mu}{d x}\right)^{((q+\varepsilon) / q)^{\prime}} d x\right)^{\varepsilon /(q+\varepsilon)} .
\end{aligned}
$$

Now we can use the reverse Hölder conditions on both $d \mu / d x$ and $|\nabla u(x)|^{q+\varepsilon}$ if $\varepsilon$ is sufficiently small (see [GM], [A] for reverse Hölder inequalities for $\nabla u$ ), to bound the last sum by

$$
\begin{array}{r}
C \sum_{Q_{j} \in \mathcal{W}}\left|Q_{j}\right|\left(\left(\frac{1}{\left|Q_{j}\right|} \int_{2 Q_{j}}|\nabla u(x)|^{2} d x\right)^{q / 2}+\left(\frac{1}{\left|Q_{j}\right|} \int_{2 Q_{j}}|\vec{f}(x)|^{q+\varepsilon}\right)^{q /(q+\varepsilon)}\right) \\
\times\left(\frac{1}{\left|Q_{j}\right|} \int_{Q_{j}}\left(\frac{d \mu}{d x}\right) d x\right) .
\end{array}
$$

Assuming that $\vec{f}$ lies in $H^{1}(\Omega)$, the Sobolev inequality allows us to replace $\left(\left|Q_{j}\right|^{-1} \int_{2 Q_{j}}|\vec{f}(x)|^{q+\varepsilon}\right)^{q /(q+\varepsilon)}$ by

$$
C\left(\left(\frac{1}{\left|Q_{j}\right|} \int_{2 Q_{j}}|\vec{f}(x)|^{2}\right)^{q / 2}+\left(\frac{1}{\left|Q_{j}\right|} \int_{2 Q_{j}}|\operatorname{div} \vec{f}(x)|^{2}\right)^{q / 2}\right) .
$$

Simplifying gives

$$
\begin{aligned}
& \int_{\Omega}|\nabla u(x)|^{q} d \mu(x) \leq C \sum_{Q_{j} \in \mathcal{W}} \mu\left(Q_{j}\right)\left(\frac{1}{\left|Q_{j}\right|} \int_{2 Q_{j}}|\nabla u(x)|^{2} d x\right)^{q / 2} \\
& \quad+C \sum_{Q_{j} \in \mathcal{W}} \mu\left(Q_{j}\right)\left(\left(\frac{1}{\left|Q_{j}\right|} \int_{2 Q_{j}}|\vec{f}(x)|^{2}\right)^{q / 2}+\left(\frac{1}{\left|Q_{j}\right|} \int_{2 Q_{j}}|\operatorname{div} \vec{f}(x)|^{2}\right)^{q / 2}\right) .
\end{aligned}
$$

By duality it will suffice to bound the three expressions:

$$
\begin{aligned}
& \sup _{\left\|\left\{g\left(Q_{j}\right)\right\}\right\|_{l^{q^{\prime}(\Omega, \mu)}}}=1 \\
& \sup _{Q_{j} \in \mathcal{W}} g\left(Q_{j}\right) \mu\left(Q_{j}\right)\left(\frac{1}{\left|Q_{j}\right|} \int_{2 Q_{j}}|\nabla u(x)|^{2} d x\right)^{1 / 2}, \\
& \sup _{\left\|\left\{g\left(Q_{j}\right)\right\}\right\|_{q^{\prime}(\Omega, \mu)}=} \sum_{Q_{j} \in \mathcal{W}} g\left(Q_{j}\right) \mu\left(Q_{j}\right)\left(\frac{1}{\left|Q_{j}\right|} \int_{2 Q_{j}}|\vec{f}(x)|^{2}\right)^{1 / 2}, \\
& \sup _{\left\|\left\{g\left(Q_{j}\right)\right\}\right\|_{l^{\prime}(\Omega, \mu)}=1} \sum_{Q_{j} \in \mathcal{W}} g\left(Q_{j}\right) \mu\left(Q_{j}\right)\left(\frac{1}{\left|Q_{j}\right|} \int_{2 Q_{j}}|\operatorname{div} \vec{f}(x)|^{2}\right)^{1 / 2} .
\end{aligned}
$$

Moreover we can assume $\left\{g\left(Q_{j}\right)\right\}$ is a finite sequence. The second and third sums are handled in the same way, so we only write out the details of bounding the second sum. By Hölder's inequality 


$$
\left(\int_{2 Q_{j}}|\vec{f}(x)|^{2}\right)^{1 / 2} \leq\left(\int_{2 Q_{j}}|\vec{f}(x)|^{p} d \nu(x)\right)^{1 / p}\left(\int_{2 Q_{j}}\left(\frac{d \nu}{d x}\right)^{-(2 / p)(p / 2)^{\prime}} d x\right)^{1 / 2(p / 2)^{\prime}}
$$

Using Hölder's inequality on the sum now gives

$$
\begin{aligned}
\sum_{Q_{j} \in \mathcal{W}} g\left(Q_{j}\right) & \mu\left(Q_{j}\right)\left(\frac{1}{\left|Q_{j}\right|} \int_{2 Q_{j}}|\vec{f}(x)|^{2}\right)^{1 / 2} \leq\left(\sum_{Q_{j} \in \mathcal{W} 2 Q_{j}}|\vec{f}(x)|^{p} d \nu(x)\right)^{1 / p} \\
& \times\left(\sum_{Q_{j} \in \mathcal{W}}\left(\frac{g\left(Q_{j}\right) \mu\left(Q_{j}\right)}{\left|Q_{j}\right|^{1 / 2}}\right)^{p^{\prime}}\left(\int_{2 Q_{j}}\left(\frac{d \nu}{d x}\right)^{-2 /(p-2)} d x\right)^{p^{\prime} / 2(p / 2)^{\prime}}\right)^{1 / p^{\prime}} .
\end{aligned}
$$

So we need to bound the second term by

$$
C\left(\sum_{Q_{j} \in \mathcal{W}} g\left(Q_{j}\right)^{q^{\prime}} \mu\left(Q_{j}\right)\right)^{1 / q^{\prime}}
$$

It is enough show that

$$
\begin{aligned}
\left(\sum_{Q_{j} \in \mathcal{W}}\left(\frac{g\left(Q_{j}\right) \mu\left(Q_{j}\right)}{\left|Q_{j}\right|^{1 / 2}}\right)^{p^{\prime}}\left(\int_{2 Q_{j}}\left(\frac{d \nu}{d x}\right)^{-2 /(p-2)} d x\right)^{p^{\prime} / 2(p / 2)^{\prime}}\right)^{q^{\prime} / p^{\prime}} \\
\leq C \sum_{Q_{j} \in \mathcal{W}} g\left(Q_{j}\right)^{q^{\prime}} \mu\left(Q_{j}\right) .
\end{aligned}
$$

Now, $p \leq q$ so $q^{\prime} \leq p^{\prime}$ and $q^{\prime} / p^{\prime} \leq 1$. Consequently, the left hand side of the last inequality is less than or equal to

$$
\sum_{Q_{j} \in \mathcal{W}}\left(\frac{g\left(Q_{j}\right) \mu\left(Q_{j}\right)}{\left|Q_{j}\right|^{1 / 2}}\right)^{q^{\prime}}\left(\int_{2 Q_{j}}\left(\frac{d \nu}{d x}\right)^{-2 /(p-2)} d x\right)^{q^{\prime} / 2(p / 2)^{\prime}} .
$$

If we compare the last two expressions term by term we will have a sufficient condition to obtain the desired inequality. So we need to have

$$
\left(\frac{g\left(Q_{j}\right) \mu\left(Q_{j}\right)}{\left|Q_{j}\right|^{1 / 2}}\right)^{q^{\prime}}\left(\int_{2 Q_{j}}\left(\frac{d \nu}{d x}\right)^{-2 /(p-2)} d x\right)^{q^{\prime} / 2(p / 2)^{\prime}} \lesssim g\left(Q_{j}\right)^{q^{\prime}} \mu\left(Q_{j}\right)
$$

or

$$
\frac{\mu\left(Q_{j}\right)^{q^{\prime}-1}}{\left|Q_{j}\right|^{q^{\prime} / 2}}\left(\int_{2 Q_{j}}\left(\frac{d \nu}{d x}\right)^{-2 /(p-2)} d x\right)^{q^{\prime} / 2(p / 2)^{\prime}} \leq C_{0}^{\prime}
$$

Taking $q^{\prime}$ th roots gives

$$
\mu\left(Q_{j}\right)^{1 / q}\left(\int_{2 Q_{j}}\left(\frac{d \nu}{d x}\right)^{-2 /(p-2)} d x\right)^{(p-2) / 2 p} \leq C_{0}\left|Q_{j}\right|^{1 / 2}
$$


This condition also implies that

$$
\begin{aligned}
\sup _{\left\|\left\{g\left(Q_{j}\right)\right\}\right\|_{l^{\prime}(\Omega, \mu)}=1} \sum_{Q_{j} \in \mathcal{W}} g\left(Q_{j}\right) \mu\left(Q_{j}\right)\left(\frac{1}{\left|Q_{j}\right|}\right. & \left.\int_{2 Q_{j}}|\operatorname{div} \vec{f}(x)|^{2}\right)^{1 / 2} \\
& \leq C\left(\int_{\Omega}|\operatorname{div} \vec{f}(x)|^{p} d \nu(x)\right)^{1 / p} .
\end{aligned}
$$

To handle the first sum,

$$
\sup _{\left\|\left\{g\left(Q_{j}\right)\right\}\right\|_{q^{\prime}(\Omega, \mu)}} \sum_{Q_{j} \in \mathcal{W}} g\left(Q_{j}\right) \mu\left(Q_{j}\right)\left(\frac{1}{\left|Q_{j}\right|} \int_{2 Q_{j}}|\nabla u(x)|^{2} d x\right)^{1 / 2},
$$

we note that

$$
\begin{aligned}
\left(\frac{1}{\left|Q_{j}\right|} \int_{2 Q_{j}}|\nabla u(x)|^{2} d x\right)^{1 / 2} \leq & \left(\frac{1}{\left|Q_{j}\right|} \int_{2 Q_{j}}|\nabla u(x)-\nabla \widetilde{u}(x)|^{2} d x\right)^{1 / 2} \\
& +\left(\frac{1}{\left|Q_{j}\right|} \int_{2 Q_{j}}|\nabla \widetilde{u}(x)|^{2} d x\right)^{1 / 2}=I+I I,
\end{aligned}
$$

with $\widetilde{u}(x)=\int_{4 Q_{j}}(\operatorname{div} \vec{f}(y)) \widetilde{G}(x, y) d y$, where $\widetilde{G}(x, y)$ is the Green function for $L$ on the domain $4 Q_{j}$. Since

$$
\left(\frac{1}{\left|Q_{j}\right|} \int_{2 Q_{j}}|\nabla \widetilde{u}(x)|^{2} d x\right)^{1 / 2} \leq C\left(\frac{1}{\left|Q_{j}\right|} \int_{4 Q_{j}}|\vec{f}(x)|^{2} d x\right)^{1 / 2}
$$

by standard results, the sum containing $I I$ can be handled as above. We are left with estimating

$$
\sup _{\left\|\left\{g\left(Q_{j}\right)\right\}\right\|_{l^{\prime}(\Omega, \mu)}} \sum_{Q_{j} \in \mathcal{W}} g\left(Q_{j}\right) \mu\left(Q_{j}\right)\left(\frac{1}{\left|Q_{j}\right|} \int_{2 Q_{j}}|\nabla(u-\widetilde{u})(x)|^{2} d x\right)^{1 / 2} .
$$

To do this, notice that

$$
\begin{aligned}
& \left(\frac{1}{\left|Q_{j}\right|} \int_{2 Q_{j}}|\nabla(u-\widetilde{u})(x)|^{2} d x\right)^{1 / 2} \\
& \quad=\left(\frac{1}{\left|Q_{j}\right|} \int_{2 Q_{j}}\left|\int_{\Omega}(\operatorname{div} \vec{f}(y))\left(\nabla_{x} G(x, y)-\nabla_{x} \widetilde{G}(x, y)\right) d y\right|^{2} d x\right)^{1 / 2} \\
& \quad \leq \int_{\Omega}|\operatorname{div} \vec{f}(y)|\left(\frac{1}{\left|Q_{j}\right|} \int_{2 Q_{j}}\left|\nabla_{x}(G(x, y)-\widetilde{G}(x, y))\right|^{2} d x\right)^{1 / 2} d y .
\end{aligned}
$$

Now for each $y \in 4 Q_{j}, G(x, y)-\widetilde{G}(x, y)$ is a solution to $L v=0$ in $\operatorname{supp}(\widetilde{G}(\cdot, y)) \subset 4 Q_{j}$ (see $[\mathrm{K}$, pp. 87-88]), and we have, by the Caccioppoli 
inequality,

$$
\begin{aligned}
\left(\frac{1}{\left|Q_{j}\right|} \int_{2 Q_{j}} \mid \nabla_{x}(G(x, y)\right. & \left.-\widetilde{G}(x, y))\left.\right|^{2} d x\right)^{1 / 2} \\
\leq & \frac{1}{l\left(Q_{j}\right)}\left(\frac{C}{\left|Q_{j}\right|} \int_{3 Q_{j}}|(G(x, y)-\widetilde{G}(x, y))|^{2} d x\right)^{1 / 2}
\end{aligned}
$$

Since $G(x, y)-\widetilde{G}(x, y) \geq 0$ by the maximum principle, we can use the Harnack inequality to obtain

$$
\left(\frac{C}{\left|Q_{j}\right|} \int_{3 Q_{j}}|G(x, y)-\widetilde{G}(x, y)|^{2} d x\right)^{1 / 2} \leq \frac{C}{\left|Q_{j}\right|} \int_{3 Q_{j}}(G(x, y)-\widetilde{G}(x, y)) d x .
$$

If $y \in \Omega \backslash 4 Q_{j}$, then $\widetilde{G}(x, y)=0$ for $x \in 2 Q_{j}$, and $G(x, y)$ is a positive solution to $L_{x} v=0$ in $3.5 Q_{j}$. In this case

$$
\begin{gathered}
\left(\frac{1}{\left|Q_{j}\right|} \int_{2 Q_{j}}\left|\nabla_{x}(G(x, y)-\widetilde{G}(x, y))\right|^{2} d x\right)^{1 / 2}=\left(\frac{1}{\left|Q_{j}\right|} \int_{2 Q_{j}}\left|\nabla_{x} G(x, y)\right|^{2} d x\right)^{1 / 2} \\
\leq \frac{1}{l\left(Q_{j}\right)}\left(\frac{C}{\left|Q_{j}\right|} \int_{3 Q_{j}}|G(x, y)|^{2} d x\right)^{1 / 2} \leq \frac{1}{l\left(Q_{j}\right)} \frac{C}{\left|Q_{j}\right|} \int_{3 Q_{j}} G(x, y) d x \\
=\frac{1}{l\left(Q_{j}\right)} \frac{C}{\left|Q_{j}\right|} \int_{3 Q_{j}}(G(x, y)-\widetilde{G}(x, y)) d x
\end{gathered}
$$

for the same reasons. Putting all this together we see that

$$
\begin{aligned}
& \sum_{Q_{j} \in \mathcal{W}} g\left(Q_{j}\right) \mu\left(Q_{j}\right)\left(\frac{1}{\left|Q_{j}\right|} \int_{2 Q_{j}}|\nabla(u-\widetilde{u})(x)|^{2} d x\right)^{1 / 2} \\
& \leq C \sum_{Q_{j} \in \mathcal{W}} g\left(Q_{j}\right) \mu\left(Q_{j}\right) \int_{\Omega}|\operatorname{div} \vec{f}(y)| \frac{1}{l\left(Q_{j}\right)}\left(\frac{1}{\left|Q_{j}\right|} \int_{3 Q_{j}}(G(x, y)-\widetilde{G}(x, y)) d x\right) d y .
\end{aligned}
$$

The last expression is then dominated by

$$
\begin{array}{r}
C \int_{\Omega}|\operatorname{div} \vec{f}(y)| \sum_{Q_{j} \in \mathcal{W}} g\left(Q_{j}\right) \mu\left(Q_{j}\right) \frac{1}{l\left(Q_{j}\right)}\left(\frac{1}{\left|Q_{j}\right|} \int_{3 Q_{j}}(G(x, y)-\widetilde{G}(x, y)) d x\right) d y \\
=C \int_{\Omega}|\operatorname{div} \vec{f}(y)| \sum_{Q_{j} \in \mathcal{W}} \lambda_{j} \varphi_{\left(Q_{j}\right)}(y) d y .
\end{array}
$$

The constants $\lambda_{j}$ are taken to be

$$
\lambda_{j}=\frac{g\left(Q_{j}\right) \mu\left(Q_{j}\right)}{l\left(Q_{j}\right) \sqrt{\left|Q_{j}\right|}}
$$


and the functions $\varphi_{\left(Q_{j}\right)}$ are defined by

$$
\varphi_{\left(Q_{j}\right)}(y)=\frac{1}{\sqrt{\left|Q_{j}\right|}} \int_{3 Q_{j}}(G(x, y)-\widetilde{G}(x, y)) d x .
$$

Assuming for now that the $\varphi_{\left(Q_{j}\right)}(y)$ satisfy (a), ( $\left.\mathrm{a}^{\prime}\right),(\mathrm{b})$, and (c), Hölder's inequality followed by the application of Theorem 1 to the function $h(y)=$ $\sum_{Q_{j} \in \mathcal{W}} \lambda_{j} \varphi_{\left(Q_{j}\right)}(y)$ gives

$$
\begin{aligned}
\int_{\Omega}|\operatorname{div} \vec{f}(y)| \sum_{Q_{j} \in \mathcal{W}} \lambda_{j} \varphi_{\left(Q_{j}\right)}(y) d y & \\
& \leq\left(\int_{\Omega}|\operatorname{div} \vec{f}(y)|^{p} d \nu(y)\right)^{1 / p}\left(\int_{\Omega}|h(y)|^{p^{\prime}} d \sigma(y)\right)^{1 / p^{\prime}} \\
& \leq C \mid \operatorname{div} \vec{f}\left\|_{L^{p}(\Omega, d \nu)}\right\| g^{*}(h) \|_{L^{p^{\prime}}(\Omega, d \sigma)} .
\end{aligned}
$$

It will suffice to show that $\left\|g^{*}(h)\right\|_{L^{p^{\prime}(\Omega, d \sigma)}}^{p^{\prime}} \leq C\left\|\left\{g\left(Q_{j}\right)\right\}\right\|_{l^{\prime}(\Omega, \mu)}^{p^{\prime}}$. Using $p>2$ implies $p^{\prime} / 2<1$. Recall that the sum defining $h(y)$ is finite. We have

$$
\begin{aligned}
\left\|g^{*}(h)\right\|_{L^{p^{\prime}}(\Omega, d \sigma)}^{p^{\prime}} & =\int_{\Omega}\left(\sum \frac{\lambda_{j}^{2}}{\left|Q_{j}\right|}\left(1+\frac{\left|y-x_{j}\right|}{l\left(Q_{j}\right)}\right)^{-n}\right)^{p^{\prime} / 2} d \sigma(y) \\
& \leq \sum \frac{\lambda_{j}^{p^{\prime}}}{\left|Q_{j}\right|^{p^{\prime} / 2}} \int_{\Omega}\left(1+\frac{\left|y-x_{j}\right|}{l\left(Q_{j}\right)}\right)^{-n p^{\prime} / 2} d \sigma(y) .
\end{aligned}
$$

So it suffices to show that the last sum is dominated by $\left(\sum g\left(Q_{j}\right)^{q^{\prime}}\right.$ $\left.\times \mu\left(Q_{j}\right)\right)^{p^{\prime} / q^{\prime}}$. Once again, taking advantage of the fact that $q^{\prime} / p^{\prime} \leq 1$, this is equivalent to showing that

$$
\sum \frac{\lambda_{j}^{q^{\prime}}}{\left|Q_{j}\right|^{q^{\prime} / 2}}\left(\int_{\Omega}\left(1+\frac{\left|y-x_{j}\right|}{l\left(Q_{j}\right)}\right)^{-n p^{\prime} / 2} d \sigma(y)\right)^{q^{\prime} / p^{\prime}} \leq \sum g\left(Q_{j}\right)^{q^{\prime}} \mu\left(Q_{j}\right) .
$$

So if

$$
\frac{\lambda_{j}^{q^{\prime}}}{\left|Q_{j}\right|^{q^{\prime} / 2}}\left(\int_{\Omega}\left(1+\frac{\left|y-x_{j}\right|}{l\left(Q_{j}\right)}\right)^{-n p^{\prime} / 2} d \sigma(y)\right)^{q^{\prime} / p^{\prime}} \leq C g\left(Q_{j}\right)^{q^{\prime}} \mu\left(Q_{j}\right)
$$

we will have the desired result. But this is the same as requiring that

$$
\mu\left(Q_{j}\right)^{1 / q}\left(\int_{\Omega}\left(1+\frac{\left|y-x_{j}\right|}{l\left(Q_{j}\right)}\right)^{-n p^{\prime} / 2} d \sigma(y)\right)^{1 / p^{\prime}} \leq C l\left(Q_{j}\right)^{n+1} .
$$

The verification of $(a),\left(a^{\prime}\right)$, and $(b)$ for the functions

$$
\varphi_{\left(Q_{j}\right)}(y)=\frac{1}{\sqrt{\left|Q_{j}\right|}} \int_{3 Q_{j}}(G(x, y)-\widetilde{G}(x, y)) d x
$$


follows directly from the estimates of Grüter and Widman [GW] for the Green function on any bounded domain satisfying an exterior cone condition. To see that (c) is also true, we may assume that $\lambda_{j} \geq 0$ and that $\varphi_{\left(Q_{j}\right)}(y) \geq 0$ on $\Omega$. We can write

$$
\begin{aligned}
\int_{\Omega}|h(y)|^{2} d y & =\int_{\Omega}\left|\sum_{Q_{j} \in \mathcal{F}} \lambda_{j} \varphi_{\left(Q_{j}\right)}(y)\right|^{2} d y=\sum_{Q_{j} \in \mathcal{F}} \lambda_{j} \int_{\Omega} h(y) \varphi_{\left(Q_{j}\right)}(y) d y \\
& =\sum_{Q_{j} \in \mathcal{F}} \lambda_{j} \int_{\Omega} h(y)\left(\frac{1}{\sqrt{\left|Q_{j}\right|}} \int_{3 Q_{j}}(G(x, y)-\widetilde{G}(x, y)) d x\right) d y \\
& \leq \sum_{Q_{j} \in \mathcal{F}} \lambda_{j} \frac{1}{\sqrt{\left|Q_{j}\right|}} \int_{3 Q_{j}} v(x) d x \\
& \leq\left(\sum_{Q_{j} \in \mathcal{F}} \lambda_{j}^{2}\right)^{1 / 2}\left(\sum_{Q_{j} \in \mathcal{F}}\left(\frac{1}{\sqrt{\left|Q_{j}\right|}} \int_{3 Q_{j}} v(x) d x\right)^{2}\right)^{1 / 2} \\
& \leq\left(\sum_{Q_{j} \in \mathcal{F}} \lambda_{j}^{2}\right)^{1 / 2}\left(\sum_{Q_{j} \in \mathcal{F} 3 Q_{j}} v(x)^{2} d x\right)^{1 / 2} \\
& \leq C\left(\sum_{Q_{j} \in \mathcal{F}} \lambda_{j}^{2}\right)^{1 / 2}\left(\int_{\Omega} v(x)^{2} d x\right)^{1 / 2} \leq C^{\prime}\left(\sum_{Q_{j} \in \mathcal{F}} \lambda_{j}^{2}\right)^{1 / 2}\left(\int_{\Omega} h(x)^{2} d x\right)^{1 / 2} .
\end{aligned}
$$

We have taken $v(x)=\int_{\Omega} G(x, y) h(y) d y$ to be the solution to $L v=h$ in $\Omega$. Dividing by $\left(\int_{\Omega} h(x)^{2} d x\right)^{1 / 2}$ gives the property of almost orthogonality.

In contrast to Theorem 2 the condition on the weights for the inequality with the local Hölder norm replacing $|\nabla u|$ is much simpler. The other obvious advantage of using Hölder norms is that one obtains results for a larger range of exponents $p$ and $q$. Suppose $\Omega$ is a bounded domain in $\mathbb{R}^{n}$ that satisfies an exterior cone condition. Then we have

THEOREM 3. Let $u$ be a solution to $L u=\operatorname{div} \vec{f}$ on $\Omega,\left.u\right|_{\partial \Omega}=0$, and let $\mu$ and $\nu$ be Borel measures on $\Omega$, with $\nu$ finite and absolutely continuous with respect to Lebesgue measure, and $d \sigma(x)=(d \nu / d x)^{1-p^{\prime}} d x$. If there is a constant $C>0$ so that

$$
\mu\left(Q_{j}\right)^{1 / q} \sup _{w \in 2 Q_{j}}\left(\int_{\Omega} \frac{1}{|w-y|^{(n+\alpha-2) p^{\prime}}} d \sigma(y)\right)^{1 / p^{\prime}} \leq C\left|Q_{j}\right|^{1 / q}
$$

for all dyadic cubes $Q_{j}$ in $\mathcal{W}$, then for any $0<q<\infty, 1<p<\infty$, there is a constant $C^{\prime}=C^{\prime}(C, n, \lambda, \alpha, q, \Omega)$ so that

$$
\left(\int_{\Omega}\|u(x)\|_{H^{\alpha}}^{q} d \mu(x)\right)^{1 / q} \leq C^{\prime}\left(\int_{\Omega}|\operatorname{div} \vec{f}(x)|^{p} d \nu(x)\right)^{1 / p},
$$

with $\|u(x)\|_{H^{\alpha}}$ defined as above, $\alpha=\alpha(n, \lambda, \partial \Omega)$. 
Proof. As in the proof of Theorem 2 we start by subdividing $\Omega$ into Whitney cubes $Q_{j}$ :

$$
\begin{aligned}
\int_{\Omega}\|u(x)\|_{H^{\alpha}}^{q} d \mu(x) & =\sum_{Q_{j} \in \mathcal{W}} \int_{Q_{j}}\|u(x)\|_{H^{\alpha}}^{q} d \mu(x) \\
& =\sum_{Q_{j} \in \mathcal{W}} \int_{Q_{j}}\left(\sup _{w \in P(x), w \neq x} \frac{|u(x)-u(w)|}{|x-w|^{\alpha}}\right)^{q} d \mu(x) \\
& \leq \sum_{Q_{j} \in \mathcal{W}} \int_{Q_{j}}\left(\sup _{\substack{x \in Q_{j}, x \neq w \\
w \in 2 Q_{j}}} \frac{|u(x)-u(w)|}{|x-w|^{\alpha}}\right)^{q} d \mu(x) \\
& =\sum_{Q_{j} \in \mathcal{W}}\left(\sup _{\substack{\sup _{j}, x \neq w \\
w \in 2 Q_{j}}} \frac{|u(x)-u(w)|}{|x-w|^{\alpha}}\right)^{q} \int_{Q_{j}} d \mu(x) .
\end{aligned}
$$

Now the integral representation of $u(x)$ and the fact that the Green function is Hölder continuous imply the last expression is dominated by

$$
\begin{gathered}
C \sum_{Q_{j} \in \mathcal{W}} \mu\left(Q_{j}\right)\left(\sup _{\substack{x \in Q_{j}, x \neq w \\
w \in 2 Q_{j}}} \frac{1}{|x-w|^{\alpha}} \int_{\Omega}|\operatorname{div} \vec{f}(y)||G(x, y)-G(w, y)| d y\right)^{q} \\
\leq C \sum_{Q_{j} \in \mathcal{W}} \mu\left(Q_{j}\right)\left(\sup _{\substack{x \in Q_{j}, x \neq w \\
w \in 2 Q_{j}}} \frac{1}{|x-w|^{\alpha}}\right. \\
\left.\times \int_{\Omega}|\operatorname{div} \vec{f}(y)||x-w|^{\alpha}\left(\frac{1}{|x-y|^{n-2+\alpha}}+\frac{1}{|w-y|^{n-2+\alpha}}\right) d y\right)^{q} \\
\leq C \sum_{Q_{j} \in \mathcal{W}} \mu\left(Q_{j}\right)\left(\sup _{w \in 2 Q_{j}} \int_{\Omega}|\operatorname{div} \vec{f}(y)||w-y|^{2-n-\alpha} d y\right)^{q} .
\end{gathered}
$$

The next to last inequality was obtained from the result of Theorem 1.9 in $[\mathrm{GW}$. The constant $C$ has changed from one line to the next, but is independent of $u, f$ and $Q_{j}$. The last sum is less than or equal to

$$
\begin{aligned}
C \sum_{Q_{j} \in \mathcal{W}} \mu\left(Q_{j}\right)\left(\int_{\Omega}|\operatorname{div} \vec{f}(x)|^{p} d \nu(x)\right)^{q / p}\left(\sup _{w \in 2 Q_{j}}\left(\int_{\Omega}|w-y|^{(2-n-\alpha) p^{\prime}} d \sigma(y)\right)^{1 / p^{\prime}}\right) \\
=C\left(\int_{\Omega}|\operatorname{div} \vec{f}(x)|^{p} d \nu(x)\right)^{q / p} \\
\sum_{Q_{j} \in \mathcal{W}} \mu\left(Q_{j}\right)\left(\sup _{w \in 2 Q_{j}}\left(\int_{\Omega}|w-y|^{(2-n-\alpha) p^{\prime}} d \sigma(y)\right)^{1 / p^{\prime}}\right) .
\end{aligned}
$$

The $q$ th root of this last expression will be bounded by

$$
C|\Omega|^{1 / q}\left(\int_{\Omega}|\operatorname{div} \vec{f}(x)|^{p} d \nu(x)\right)^{1 / p}
$$


if

$$
\mu\left(Q_{j}\right)^{1 / q} \sup _{w \in 2 Q_{j}}\left(\int_{\Omega}|w-y|^{(2-n-\alpha) p^{\prime}} d \sigma(y)\right)^{1 / p^{\prime}} \leq\left|Q_{j}\right|^{1 / q}
$$

for every $Q_{j} \in \mathcal{W}$.

3. Proof of Theorem 1. To prove Theorem 1 we follow the method of Wilson [W] in using the functions

$$
F(I, x)=\sum_{J \in \mathcal{S}(I)} \lambda_{J} \varphi_{(J)}(x), \quad F(I)=F\left(I, x_{I}\right), \quad F^{*}(x)=\sup _{I \ni x} F(I)
$$

and

$$
\begin{aligned}
G(I, x) & =\left(\sum_{J \in \mathcal{S}(I)} \frac{\lambda_{J}^{2}}{|J|}\left(1+\frac{\left|x-x_{J}\right|}{l(J)}\right)^{-n}\right)^{1 / 2}, \\
G(I) & =G\left(I, x_{I}\right), \quad G^{*}(x)=\sup _{I \ni x} G(I) .
\end{aligned}
$$

They are always generated by a given function $f(x)=\sum_{J \in \mathcal{F}} \lambda_{J} \varphi_{(J)}(x)$, where $\mathcal{F}$ is a finite family of dyadic cubes, $\mathcal{S}(I)=\{J \in \mathcal{F}: J \not \subset I\}$, and $l(I)$ is the side length of the dyadic cube $I . F(I, x)$ and $G(I, x)$ are only defined for $x \in I$. We note some special properties of the particular functions $\varphi_{(J)}(x)$ that were used in the proof of Theorem 2. These properties will be crucial in proving the estimates in Lemmas 1-7 and the Central Lemma. We have $\varphi_{(J)}(x)=0$ whenever $x$ lies outside $\Omega$. Also each $\varphi_{(J)}$ is chosen so that $J \in \mathcal{W}$. As in $[\mathrm{W}]$ we obtain local estimates relating the functions $F(I, x)$, $G(I, x)$, etc. in order to use these functions to prove the crucial good- $\lambda$ inequality of the Corollary to the Central Lemma. The good- $\lambda$ inequality then yields the result of Theorem 1 by standard methods. The local estimates are established in Lemmas 1-7 below.

For the remaining part of the paper we take $f(x)=\sum_{J \in \mathcal{F}} \lambda_{J} \varphi_{(J)}(x)$, where $\mathcal{F}$ is a finite family of dyadic cubes; the $\varphi_{(J)}$ satisfy properties (a), $\left(\mathrm{a}^{\prime}\right),(\mathrm{b})$, and (c), and they have all the properties mentioned in the previous paragraph. We note that many of the constants obtained in Lemmas 1-7 depend on $\operatorname{diam}(\Omega)$. For the functions $\varphi_{(J)}$ that appeared in the proof of Theorem 2, i.e.

$$
\varphi_{(J)}(y)=\frac{1}{\sqrt{|J|}}\left(\int_{2 J}(G(x, y)-\widetilde{G}(x, y)) d x\right)
$$

the constants in $\left(\mathrm{a}^{\prime}\right)$ and (b) also depend on $\operatorname{diam}(\Omega)$ and $\Omega$ (see $[\mathrm{GW}]$ ), so this is no new restriction. We also note that having $\varphi_{(J)}(x)=0$ whenever $x$ lies outside $\Omega$ means that $F(I, x)=0$ when $x \in \Omega^{\mathrm{c}}$. However, $F^{*}(x)$, $G(I, x), G^{*}(x)$ are not necessarily zero for $x$ outside $\Omega$. Following Wilson [W] we start with 
Lemma 1. $f(x) \leq F^{*}(x)$ for a.e. $x \in Q_{0}$.

Proof. This follows from the definition of $F^{*}(x)$, the fact that $\mathcal{F}$ is a finite family, and that (a) and (b) imply that $f$ is continuous.

Lemma 2. There is a constant $C$ so that $G^{*}(x) \leq C g^{*}(x)$.

Proof. We have $G^{*}(x)=\sup _{Q \ni x} G(Q)$. If $x \in Q$ and $I \in \mathcal{S}(Q)$, then either $I \supsetneq Q$ or $I$ lies outside $Q$. In both cases, $\left|x_{I}-x_{Q}\right| \geq \operatorname{cl}(Q)$ and $\left|x-x_{Q}\right| \leq c^{\prime} l(Q)$. Therefore $\left|x-x_{I}\right| \leq\left|x-x_{Q}\right|+\left|x_{Q}-x_{I}\right| \leq C\left|x_{Q}-x_{I}\right|$. So $1+\left|x-x_{I}\right| / l(I) \leq C^{\prime}\left(1+\left|x_{Q}-x_{I}\right| / l(I)\right)$ or

$$
\left(1+\left|x_{Q}-x_{I}\right| / l(I)\right)^{-n} \leq C^{\prime \prime}\left(1+\left|x-x_{I}\right| / l(I)\right)^{-n} .
$$

For $I \in \mathcal{F}$, whenever the term on the left is in $G(Q)$, the term on the right appears in $g^{*}(x), x \in Q$, multiplied by $1 / C^{\prime \prime}$. This is true for all dyadic cubes $Q$ with the same constant $C=\max \left(1, C^{\prime \prime}\right)$, so $G(Q) \leq C g^{*}(x)$.

Lemma 3. For any $0<\eta<1$, if $x \in \eta Q$, then there is a constant $C_{1}=C(n, \eta)$ so that $C_{1}^{-1} G(Q) \leq G(Q, x) \leq C_{1} G(Q)$.

Proof. For any cube $I \in \mathcal{S}(Q),\left|x-x_{I}\right| /\left|x_{Q}-x_{I}\right|$ is bounded above and below by constants that depend on $\eta$ and $n$.

Lemma 4. For any $0<\eta<1$, if $x, y \in \eta Q$, then there is a constant $C_{2}=C(n, \lambda, \eta, \operatorname{diam}(\Omega), \Omega, \alpha)$ so that $|F(Q, x)-F(Q, y)| \leq C_{2} G(Q)$.

Proof. As in $[\mathrm{W}]$ we write

$$
\begin{aligned}
|F(Q, x)-F(Q, y)|= & \left|\sum_{J \in \mathcal{S}(Q)} \lambda_{J}\left(\varphi_{(J)}(x)-\varphi_{(J)}(y)\right)\right| \\
\leq & \sum_{J \in \mathcal{S}(Q), l(J) \geq l(Q)}\left|\lambda_{J}\right|\left|\varphi_{(J)}(x)-\varphi_{(J)}(y)\right| \\
& +\sum_{J \in \mathcal{S}(Q), l(J)<l(Q)}\left|\lambda_{J}\right|\left|\varphi_{(J)}(x)-\varphi_{(J)}(y)\right|=I+I I .
\end{aligned}
$$

When $x$ and $y$ both lie inside $\Omega, I$ will be shown to be bounded by $C G(Q)$ using the Hölder continuity of the $\varphi_{(J)}$ 's (property (b)), and II should be bounded using Hölder continuity. When both $x$ and $y$ lie outside $\Omega, F(Q, x)$ and $F(Q, y)$ are both 0 , so the estimate of Lemma 4 is trivially valid. However, the situation when $x \in \Omega$ but $y \in \Omega^{\mathrm{c}}$ needs to be considered separately. We are not guaranteed that (b) is valid when one point, $x$ or $y$, lies outside the domain $\Omega$. In this case $I$ and $I I$ should be estimated using $\left(\mathrm{a}^{\prime}\right)$.

We start with the proof for $x, y \in \eta Q \subset \Omega$. Then by (b) and the CauchySchwarz inequality (remember that $n \geq 3$ ), 


$$
\begin{aligned}
& I=\sum_{J \in \mathcal{S}(Q), l(J) \geq l(Q)}\left|\lambda_{J}\right|\left|\varphi_{(J)}(x)-\varphi_{(J)}(y)\right| \\
& \lesssim \sum_{J \in \mathcal{S}(Q), l(J) \geq l(Q)}\left|\lambda_{J}\right||x-y|^{\alpha} l(J)^{2-n / 2-\alpha}\left(1+\frac{\left|x-x_{J}\right|}{l(J)}+\frac{\left|y-x_{J}\right|}{l(J)}\right)^{2-n-\alpha} \\
& \lesssim \sum_{J \in \mathcal{S}(Q), l(J) \geq l(Q)}\left|\lambda_{J}\right|\left(\frac{|x-y|}{l(J)}\right)^{\alpha} l(J)^{2-n / 2}\left(1+\frac{\left|x-x_{J}\right|}{l(J)}+\frac{\left|y-x_{J}\right|}{l(J)}\right)^{2-n-\alpha} \\
& \lesssim\left(\sum_{J \in \mathcal{S}(Q), l(J) \geq l(Q)} \frac{\lambda_{J}^{2}}{|J|}\left(1+\frac{\left|x-x_{J}\right|}{l(J)}\right)^{-n}\right)^{1 / 2} \\
& \times\left(\sum_{J \in \mathcal{S}(Q), l(J) \geq l(Q)}\left(\frac{|x-y|}{l(J)}\right)^{2 \alpha} l(J)^{4}\left(1+\frac{\left|x-x_{J}\right|}{l(J)}\right)^{4-n-2 \alpha}\right)^{1 / 2} \\
& \lesssim G(Q, x) \cdot C(d(\Omega))^{2} \\
& \times\left(\sum_{J \in \mathcal{S}(Q), l(J) \geq l(Q)}\left(\frac{|x-y|}{l(J)}\right)^{2 \alpha}\left(1+\frac{\left|x-x_{J}\right|}{l(J)}\right)^{-n-2 \alpha}\right)^{1 / 2}
\end{aligned}
$$

The last inequality follows from the fact that $l(J)\left(1+\left|x-x_{J}\right| / l(J)\right) \leq$ $c \operatorname{diam}(\Omega)=c d(\Omega)$. From Lemma $3, G(Q, x) \leq C(n, \eta) G(Q)$ because $x \in$ $\eta Q$. So we need only show that

$$
\left(\sum_{J \in \mathcal{S}(Q), l(J) \geq l(Q)}\left(\frac{|x-y|}{l(J)}\right)^{2 \alpha}\left(1+\frac{\left|x-x_{J}\right|}{l(J)}\right)^{-n-2 \alpha}\right) \leq C .
$$

Since $x$ and $y$ lie in $\eta Q$, the sum on the left can be written as

$$
\begin{aligned}
& \sum_{k=0}^{\infty} \sum_{\substack{l(J)=2^{k} l(Q) \\
J \in \mathcal{S}(Q)}} 2^{-2 \alpha k}\left(1+\frac{\left|x-x_{J}\right|}{l(J)}\right)^{-n-2 \alpha} \\
& =\sum_{k=0}^{\infty} 2^{-2 \alpha k} \sum_{j \geq k} \sum_{\substack{2^{j-1} l(Q)<l(J)+\left|x-x_{J}\right| \leq 2^{j} l(Q) \\
l(J)=2^{k} l(Q), J \in \mathcal{S}(Q)}}\left(1+\frac{\left|x-x_{J}\right|}{l(J)}\right)^{-n-2 \alpha} \\
& \leq \sum_{k=0}^{\infty} 2^{-2 \alpha k} \sum_{\substack{j \geq k \\
2^{j-k-1}<1+\left|x-x_{J}\right| / l(J) \leq 2^{j-k} \\
l(J)=2^{k} l(Q), J \in \mathcal{S}(Q)}}\left(1+\frac{\left|x-x_{J}\right|}{l(J)}\right)^{-n-2 \alpha} \\
& \leq C(n) \sum_{k=0}^{\infty} 2^{-2 \alpha k} \sum_{j=k}^{\infty} 2^{n(j-k)}\left(1+\frac{\left|x-x_{J}\right|}{l(J)}\right)^{-n-2 \alpha} .
\end{aligned}
$$


The last estimate follows from counting the number of cubes $J$ of side length $2^{k} l(Q)$ that can exist in the annular region $2^{j-k-1}<1+\left|x_{Q}-x_{J}\right| / l(J) \leq$ $2^{j-k}$ if $j \geq k$. It is easy to see that the last expression is bounded by

$$
\begin{aligned}
& C(n) \sum_{k=0}^{\infty} 2^{-2 \alpha k} \sum_{j=k}^{\infty} 2^{n(j-k)}\left(2^{j-k-1}\right)^{-(n+2 \alpha)} \leq C(n, \alpha) \sum_{k=0}^{\infty} \sum_{j=k}^{\infty} 2^{-2 \alpha j} \\
& \leq C(n, \alpha) \sum_{k=0}^{\infty} 2^{-2 \alpha k} \sum_{j=0}^{\infty} 2^{-2 \alpha j} \leq C(n, \alpha) .
\end{aligned}
$$

Now to bound $I I$, still keeping $x, y \in \eta Q \subset \Omega$, we have

$$
I I \leq \sum_{J \in \mathcal{S}(Q), l(J)<l(Q)}\left|\lambda_{J}\right|\left|\varphi_{(J)}(x)-\varphi_{(J)}(y)\right|,
$$

so by Lemma 3 it is enough to show this sum is $\leq C G(Q, x)$. Using (b) gives

$$
\sum_{J \in \mathcal{S}(Q), l(J)<l(Q)}\left|\lambda_{J}\right|\left|\varphi_{(J)}(x)-\varphi_{(J)}(y)\right|
$$

$\lesssim \sum_{J \in \mathcal{S}(Q), l(J)<l(Q)}\left|\lambda_{J}\right||x-y|^{\alpha} l(J)^{2-n / 2-\alpha}\left(1+\frac{\left|x-x_{J}\right|}{l(J)}+\frac{\left|y-x_{J}\right|}{l(J)}\right)^{2-n-\alpha}$

$\lesssim\left(\sum_{J \in \mathcal{S}(Q)} \frac{\lambda_{J}^{2}}{|J|}\left(1+\frac{\left|x-x_{J}\right|}{l(J)}\right)^{-n}\right)^{1 / 2}$

$\times\left(\sum_{J \in \mathcal{S}(Q), l(J)<l(Q)} l(Q)^{2 \alpha} l(J)^{4-2 \alpha}\left(1+\frac{\left|x-x_{J}\right|}{l(J)}\right)^{4-n-2 \alpha}\right)^{1 / 2}$

$=C G(Q, x)\left(\sum_{J \in \mathcal{S}(Q), l(J)<l(Q)}\left(\frac{l(Q)}{l(J)}\right)^{2 \alpha} l(J)^{4}\left(1+\frac{\left|x-x_{J}\right|}{l(J)}\right)^{4-n-2 \alpha}\right)^{1 / 2}$

$\leq C G(Q) \cdot H_{(Q)}(x)$.

Now,

$$
\begin{aligned}
H_{Q}(x) \leq & C(\operatorname{diam} \Omega)^{2}\left(\sum_{J \in \mathcal{S}(Q), l(J)<l(Q)}\left(\frac{l(Q)}{l(J)}\right)^{2 \alpha}\left(1+\frac{\left|x-x_{J}\right|}{l(J)}\right)^{-n-2 \alpha}\right)^{1 / 2} \\
\leq & C(d(\Omega), n, \eta) \\
& \times\left(\sum_{J \in \mathcal{S}(Q), l(J)<l(Q)}\left(\frac{l(Q)}{l(J)}\right)^{-n-2 \alpha+2 \alpha}\left(1+\frac{\left|x_{Q}-x_{J}\right|}{l(Q)}\right)^{-n-2 \alpha}\right)^{1 / 2} .
\end{aligned}
$$

The last inequality follows from the fact that for $J \cap Q=\emptyset, J \in \mathcal{S}(Q)$, $x \in \eta Q$, we have $\left|x-x_{J}\right| /\left|x_{Q}-x_{J}\right| \sim C$, and since $\left|x_{Q}-x_{J}\right| \gtrsim l(Q)$, we 
have

$$
1+\frac{\left|x-x_{J}\right|}{l(J)} \gtrsim \frac{l(Q)}{l(J)} \frac{\left|x_{Q}-x_{J}\right|}{l(Q)} \gtrsim \frac{l(Q)}{l(J)}\left(1+\frac{\left|x_{Q}-x_{J}\right|}{l(Q)}\right) .
$$

This means that

$$
\left(1+\frac{\left|x-x_{J}\right|}{l(J)}\right)^{-n-2 \alpha} \lesssim\left(\frac{l(Q)}{l(J)}\left(1+\frac{\left|x_{Q}-x_{J}\right|}{l(Q)}\right)\right)^{-n-2 \alpha}
$$

Finally, to estimate

$$
\left(\sum_{J \in \mathcal{S}(Q), l(J)<l(Q)}\left(\frac{l(Q)}{l(J)}\right)^{-n}\left(1+\frac{\left|x_{Q}-x_{J}\right|}{l(Q)}\right)^{-n-2 \alpha}\right)^{1 / 2}
$$

we can proceed as in [W] to divide $Q_{0} \backslash Q$ into dyadic cubes $Q^{\prime}$ whose size is the same as that of $Q$. We write the sum as

$$
\begin{aligned}
& \left(\sum_{Q^{\prime} \subset Q_{0} \backslash Q} \sum_{J \in \mathcal{S}(Q), J \subset Q^{\prime}}\left(\frac{l(J)}{l(Q)}\right)^{n}\left(1+\frac{\left|x_{Q}-x_{J}\right|}{l(Q)}\right)^{-n-2 \alpha}\right)^{1 / 2} \\
& \lesssim\left(\sum_{Q^{\prime} \subset Q_{0} \backslash Q} \sum_{J \in \mathcal{S}(Q), J \subset Q^{\prime}}\left(\frac{l(J)}{l(Q)}\right)^{n}\left(1+\frac{\left|x_{Q}-x_{Q^{\prime}}\right|}{l\left(Q^{\prime}\right)}\right)^{-n-2 \alpha}\right)^{1 / 2},
\end{aligned}
$$

which is valid since $\left|x_{Q}-x_{J}\right| \gtrsim\left|x_{Q}-x_{Q^{\prime}}\right|$. Now the $J$ are Whitney cubes from $\mathcal{F}$, so they are disjoint. Consequently, for each $Q^{\prime}$,

$$
\sum_{J \subset Q^{\prime}}\left(\frac{l(J)}{l(Q)}\right)^{n}=\sum_{J \subset Q^{\prime}} \frac{|J|}{\left|Q^{\prime}\right|} \leq 1
$$

Therefore we can write

$$
\begin{aligned}
H_{Q}(x)^{2} & \lesssim \sum_{k=0}^{\infty} \sum_{\substack{Q^{\prime} \subset Q_{0} \backslash Q \\
\leq\left|x_{Q}-x_{Q^{\prime}}\right|<2^{k} l(Q)}}\left(1+\frac{\left|x_{Q}-x_{Q^{\prime}}\right|}{l\left(Q^{\prime}\right)}\right)^{-n-2 \alpha} \\
& \lesssim \sum_{k=0}^{\infty} 2^{k n} 2^{-k(n+2 \alpha)} \leq C(\alpha, n)
\end{aligned}
$$

by counting the maximum number of cubes $Q^{\prime}$ that can lie inside the annular region $2^{k-1} l(Q) \leq\left|x_{Q}-x_{Q^{\prime}}\right|<2^{k} l(Q)$.

We have shown that $|F(Q, x)-F(Q, y)| \leq C_{2} G(Q)$ when both $x$ and $y$ lie inside $\Omega$, or when both points lie in $Q_{0} \backslash \Omega$. The remaining case is for one point lying inside $\Omega$ and the other outside $\Omega$. This implies of course that the dyadic cube $Q$ is such that $\eta Q \cap \Omega \neq \emptyset$ and $\eta Q \cap \Omega^{\mathrm{c}} \neq \emptyset$. Without loss of generality $x \in \Omega$ and $y \notin \Omega$. So $F(Q, y)=0$. Here we cannot use (b), since the decay in (b) is not necessarily valid for points outside $\Omega$. However, we 
note that $\left(\mathrm{a}^{\prime}\right)$ is useful. Since $Q$ overlaps the boundary of $\Omega$, and $x \in Q \cap \Omega$, we have $\delta(x)=\operatorname{dist}(x, \partial \Omega) \lesssim l(Q)$. So

$$
\begin{aligned}
& |F(Q, x)-F(Q, y)|=|F(Q, x)| \\
& \leq \sum_{J \in \mathcal{S}(Q), l(J) \geq l(Q)}\left|\lambda_{J}\right|\left|\varphi_{J}(x)\right|+\sum_{J \in \mathcal{S}(Q), l(J)<l(Q)}\left|\lambda_{J}\right|\left|\varphi_{J}(x)\right|=I^{\prime}+I I^{\prime} .
\end{aligned}
$$

Now,

$$
\left(I^{\prime}\right)^{2} \lesssim \sum_{J \in \mathcal{S}(Q), l(J) \geq l(Q)}\left|\lambda_{J}\right| \delta(x)^{\alpha} l(J)^{2-n / 2-\alpha}\left(1+\frac{\left|x-x_{J}\right|}{l(J)}\right)^{2-n-\alpha}
$$

from using $\left(\mathrm{a}^{\prime}\right)$ on the $\left|\varphi_{J}(x)\right|$ 's in $I^{\prime}$. The last sum is bounded by

$$
\begin{aligned}
C(\operatorname{diam}(\Omega))^{2} & \sum_{J \in \mathcal{S}(Q), l(J) \geq l(Q)}\left|\lambda_{J}\right|\left(\frac{l(Q)}{l(J)}\right)^{\alpha} l(J)^{-n / 2}\left(1+\frac{\left|x-x_{J}\right|}{l(J)}\right)^{-n-\alpha} \\
\leq & C(d(\Omega), n, \alpha, \lambda, \eta) G(Q) \\
& \times\left(\sum_{J \in \mathcal{S}(Q), l(J) \geq l(Q)}\left(\frac{l(Q)}{l(J)}\right)^{2 \alpha}\left(1+\frac{\left|x-x_{J}\right|}{l(J)}\right)^{-n-2 \alpha}\right)^{1 / 2} .
\end{aligned}
$$

Dominating the last sum by a constant follows as before. Estimating $I I^{\prime}$ follows from almost the same proof that gave the bound for $I I$ in the first case, in which $x$ and $y$ were both located inside $\Omega$. Here the fact that $\delta(x) \lesssim l(Q)$ replaces the similar estimate for $|x-y|$ in (A). After that the calculations are identical.

For the next four lemmas we define

$$
N(I)=\left\{I^{*} \in \mathfrak{D}: I^{*} \subset I \text { and } l\left(I^{*}\right)=0.5 l(I)\right\}
$$

for any dyadic cube $I \in \mathfrak{D}$. We have

Lemma 5. $G(I) \leq C G\left(I^{*}\right)$.

Proof. We have $x_{I^{*}} \in \eta I$ if $0<\eta<1$ is sufficiently large, depending on $n$. By Lemma $3, G(I) \leq C G\left(I, x_{I^{*}}\right)$, and by definition $G\left(I, x_{I^{*}}\right) \leq$ $G\left(I^{*}, x_{I^{*}}\right)=G\left(I^{*}\right)$.

Lemma 6. For $I^{*} \in N(I), G\left(I^{*}\right) \leq C G^{*}(x)$ whenever $x \in I$.

Proof. By definition $G^{*}(x)=\sup _{J \ni x} G(J)$, so if $x \in I^{*}$, then $G\left(I^{*}\right) \leq$ $G^{*}(x)$. Suppose that $x$ lies in $I \backslash I^{*}$. For any $J \subset I \backslash I^{*}$ such that $x \in J$, we have $G\left(I^{*}\right)^{2} \leq C G(J)^{2}+B$, where

$$
B=\sum_{K \subset J, K \in \mathcal{S}\left(I^{*}\right)} \frac{\lambda_{K}^{2}}{|K|}\left(1+\frac{\left|x_{I^{*}}-x_{K}\right|}{l(K)}\right)^{-n} .
$$


All the terms in $B$ occur in $G\left(I^{*}\right)^{2}$. If $L \in \mathcal{S}\left(I^{*}\right) \backslash\left\{K \subset J: K \in \mathcal{S}\left(I^{*}\right)\right\}$, then $L \in \mathcal{S}(J)$. Moreover $\left|x_{L}-x_{J}\right| \leq\left|x_{L}-x_{I^{*}}\right|+\left|x_{J}-x_{I^{*}}\right| \leq\left|x_{L}-x_{I^{*}}\right|+\operatorname{cl}(I) \leq$ $c^{\prime}\left|x_{L}-x_{I^{*}}\right|$ since $\left|x_{L}-x_{I^{*}}\right| \gtrsim l(I)$. We may assume $c^{\prime} \geq 1$; this implies that

$$
\left(1+\frac{\left|x_{L}-x_{J}\right|}{l(L)}\right)^{-n} \geq C^{\prime}\left(1+\frac{\left|x_{L}-x_{I^{*}}\right|}{l(L)}\right)^{-n} .
$$

So each term in $G\left(I^{*}\right)$ that does not occur in $B$ is less than or equal to a constant times a term that occurs in $G(J)$. Now $\mathcal{F}$ is a finite family, so for $|J|$ sufficiently small, the sum in $B$ will be empty, and $G(J) \leq G^{*}(x)$.

Lemma 7. $\left|F\left(I^{*}\right)-F(I)\right| \leq C G\left(I^{*}\right)$.

Proof. Lemmas 4 and 5 imply that $\left|F\left(I, x_{I^{*}}\right)-F(I)\right| \leq C G(I) \leq$ $C^{\prime} G\left(I^{*}\right)$; consequently, it is enough to show that $\left|F\left(I, x_{I^{*}}\right)-F\left(I^{*}\right)\right| \leq$ $C G\left(I^{*}\right)$. If $x_{I^{*}} \in \Omega^{\mathrm{c}}$, then both functions on the left are zero, so we can assume that $x_{I^{*}} \in \Omega$. We have

$$
\begin{aligned}
\left|F\left(I, x_{I^{*}}\right)-F\left(I^{*}\right)\right| & =\left|\sum_{J \in \mathcal{S}\left(I^{*}\right) \backslash \mathcal{S}(I)} \lambda_{J} \varphi_{J}\left(x_{I^{*}}\right)\right| \\
& \leq \sum_{J \in \mathcal{S}\left(I^{*}\right) \backslash \mathcal{S}(I)}\left|\lambda_{J}\right| \cdot l(J)^{2-n / 2} \cdot\left(1+\frac{\left|x_{I^{*}}-x_{J}\right|}{l(J)}\right)^{2-n}
\end{aligned}
$$

from (a). The Cauchy-Schwarz inequality gives

$$
\begin{aligned}
&\left|F\left(I, x_{I^{*}}\right)-F\left(I^{*}\right)\right| \leq\left(\sum_{J \in \mathcal{S}\left(I^{*}\right) \backslash \mathcal{S}(I)} \frac{\left|\lambda_{J}\right|^{2}}{|J|}\left(1+\frac{\left|x_{I^{*}}-x_{J}\right|}{l(J)}\right)^{-n}\right)^{1 / 2} \\
& \times\left(\sum_{J \in \mathcal{S}\left(I^{*}\right) \backslash \mathcal{S}(I)} l(J)^{4}\left(1+\frac{\left|x_{I^{*}}-x_{J}\right|}{l(J)}\right)^{4-n}\right)^{1 / 2} \\
& \leq C G\left(I^{*}\right) \cdot C(\operatorname{diam}(\Omega))^{2} \cdot\left(\sum_{J \in \mathcal{S}\left(I^{*}\right) \backslash \mathcal{S}(I)}\left(1+\frac{\left|x_{I^{*}}-x_{J}\right|}{l(J)}\right)^{-n}\right)^{1 / 2} .
\end{aligned}
$$

If we can show that $\left(\sum_{J \in \mathcal{S}\left(I^{*}\right) \backslash \mathcal{S}(I)}\left(1+\left|x_{I^{*}}-x_{J}\right| / l(J)\right)^{-n}\right)^{1 / 2}$ is bounded by a constant, we will be done. Notice that

$$
1+\frac{\left|x_{I^{*}}-x_{J}\right|}{l(J)} \geq \frac{\left|x_{I^{*}}-x_{J}\right|}{l(J)}=\frac{l(I)}{l(J)} \cdot \frac{\left|x_{I^{*}}-x_{J}\right|}{l(I)},
$$

and $\left|x_{I^{*}}-x_{J}\right| \sim l(I)$ because $J \in \mathcal{S}\left(I^{*}\right) \backslash \mathcal{S}(I)$. So

$$
\frac{l(I)}{l(J)} \cdot \frac{\left|x_{I^{*}}-x_{J}\right|}{l(I)} \geq C \frac{l(I)}{l(J)}\left(1+\frac{\left|x_{I^{*}}-x_{J}\right|}{l(I)}\right) .
$$


We have

$$
\left(1+\frac{\left|x_{I^{*}}-x_{J}\right|}{l(J)}\right)^{-n} \leq C\left(\frac{l(J)}{l(I)}\right)^{n}\left(1+\frac{\left|x_{I^{*}}-x_{J}\right|}{l(I)}\right)^{-n} .
$$

This gives

$\sum_{J \in \mathcal{S}\left(I^{*}\right) \backslash \mathcal{S}(I)}\left(1+\frac{\left|x_{I^{*}}-x_{J}\right|}{l(J)}\right)^{-n} \leq C \sum_{J \in \mathcal{S}\left(I^{*}\right) \backslash \mathcal{S}(I)}\left(\frac{l(J)}{l(I)}\right)^{n}\left(1+\frac{\left|x_{I^{*}}-x_{J}\right|}{l(I)}\right)^{-n}$. Now remember that the cubes $J$ originally came from $\mathcal{F}$ so they are disjoint. Also $J \in \mathcal{S}\left(I^{*}\right) \backslash \mathcal{S}(I)$ means that either $J=I$ or $J \subset I \backslash I^{*}$. As a result

$$
\sum_{J \in \mathcal{S}\left(I^{*}\right) \backslash \mathcal{S}(I)}\left(\frac{l(J)}{l(I)}\right)^{n}\left(1+\frac{\left|x_{I^{*}}-x_{J}\right|}{l(I)}\right)^{-n} \leq \sum_{J \in \mathcal{S}\left(I^{*}\right) \backslash \mathcal{S}(I)}\left(\frac{l(J)}{l(I)}\right)^{n} \leq 1 .
$$

The purpose of establishing Lemmas $1-7$ is to prove the following

Central Lemma. Let $f(x)=\sum_{I \in \mathcal{F}} \lambda_{I} \varphi_{(I)}(x)$, where $\mathcal{F}$ is a finite family of cubes from $\mathcal{W}$, the $\varphi_{(I)}$ satisfy $(\mathrm{a}),\left(\mathrm{a}^{\prime}\right)$, (b) and (c), and $\lambda_{I}=0$ for any $I \not \subset I_{0}$, where $I_{0}$ is a fixed cube in $\mathfrak{D}$. For any $0<\beta<1$, there is a $\gamma=\gamma(\beta, n, \lambda, \alpha, \Omega, \eta)$ such that

$$
\mid\left\{x \in I_{0}: F^{*}(x)>1 \text { and } G^{*}(x) \leq \gamma\right\}|\leq \beta| I_{0} \mid .
$$

Proof. Let $I_{j}$ be the dyadic cubes for which one of the subcubes $I_{j}^{*} \in$ $N\left(I_{j}\right)$ is a maximal dyadic cube in $I_{0}$ so that $G\left(I_{j}^{*}\right)>A \gamma$ for $A$ large enough so $A C^{-1}>1, C$ being the constant in Lemma 6 . Notice that $G\left(I_{0}\right)=0$ (and so $F\left(I_{0}, x\right)=0$ for any $\left.x \in I_{0}\right)$, and $I_{j} \subset I_{0}$. We see that $G\left(I_{j}\right) \leq A \gamma, x \in I_{j}$ implies that $G^{*}(x)>A C^{-1} \gamma>\gamma$ from Lemma 6 , and $G^{*}(x) \leq A \gamma$ for all $x \in I_{0} \backslash \bigcup I_{j}$.

Let $E=\left\{x \in I_{0}: F^{*}(x)>1\right.$ and $\left.G^{*}(x) \leq \gamma\right\}$. For any $x \in E$ there is a maximal dyadic cube $Q_{i}$ such that $F\left(Q_{i}\right)>1$. We have $Q_{i} \subset I_{0}$ and $Q_{i} \not \subset I_{j}$ for any of the maximal cubes defined in the previous paragraph, because $G^{*}(x) \leq \gamma$ means that $x$ cannot lie in $I_{j}$. Following the argument in the proof of the Main Lemma in [W], we create the family $\mathcal{G}=\left\{P_{k}\right\}$ of dyadic cubes which consists of the maximal disjoint cubes that result from combining the $I_{j}$ and the $Q_{i}$. So $E \subset \bigcup_{k} P_{k}$. In fact $x \in E$ implies that $x \in P_{k^{\prime}}$ for some maximal cube in $\mathcal{G}$ for which $F\left(P_{k^{\prime}}\right)>1$. It is also true that $G\left(P_{k^{\prime}}\right) \leq \gamma$, since $G^{*}(x) \leq \gamma$. We proceed to divide the cubes in $\mathcal{F}$ into two sets, $\mathcal{F}_{1}=\left\{J: J \not \subset P_{k}\right.$ for any $\left.P_{k} \in \mathcal{G}\right\}$ and $\mathcal{F}_{2}=\left\{J: J \subset P_{k}\right.$ for some $\left.P_{k} \in \mathcal{G}\right\}$. Writing $f(x)=\sum_{J \in \mathcal{F}_{1}} \lambda_{J} \varphi_{(J)}(x)+\sum_{J \in \mathcal{F}_{2}} \lambda_{J} \varphi_{(J)}(x)=$ $f_{1}(x)+f_{2}(x)$, we can define $F_{i}(Q, x), F_{i}^{*}(Q), G_{i}(Q, x), G_{i}^{*}(x)$ for $i=1,2$ just as we did for $f(x)$. We have $F(Q, x)=F_{1}(Q, x)+F_{2}(Q, x)$, while $G_{i}(Q, x) \leq G_{1}(Q, x)+G_{2}(Q, x)=G(Q, x)$.

The facts that $E \subset \bigcup P_{k^{\prime}}$ and that Lebesgue measure is a doubling measure mean $|E| \leq C(n) \sum_{k^{\prime}}\left|c\left(P_{k^{\prime}}\right)\right|$, where $c\left(P_{k}\right)=\left\{x \in P_{k}: x \in \frac{1}{10} P_{k}\right\}$. 
For $x \in P_{k^{\prime}}$, we must have either $F_{1}\left(P_{k^{\prime}}\right)>0.5$ or $F_{2}\left(P_{k^{\prime}}\right)>0.5$. For $x \in c\left(P_{k^{\prime}}\right)$, Lemma 4 says that either $F_{1}\left(P_{k^{\prime}}, x\right)>0.25$ or $F_{2}\left(P_{k^{\prime}}, x\right)>0.25$ whenever $\gamma$ is small enough. Also

$$
\begin{aligned}
& \sum_{k^{\prime}}\left|c\left(P_{k^{\prime}}\right)\right| \leq \sum_{F_{1}\left(P_{k^{\prime}}\right)>0.5}\left|c\left(P_{k^{\prime}}\right)\right|+\sum_{F_{2}\left(P_{k^{\prime}}\right)>0.5}\left|c\left(P_{k^{\prime}}\right)\right| \\
& \leq \sum_{k}\left|\left\{x \in c\left(P_{k}\right): F_{1}\left(P_{k}, x\right)>0.25\right\}\right|+\sum_{k}\left|\left\{x \in c\left(P_{k}\right): F_{2}\left(P_{k}, x\right)>0.25\right\}\right| .
\end{aligned}
$$

Using Chebyshev's inequality we can say we only need to estimate

$$
\sum_{k} 16 \int_{c\left(P_{k}\right)}\left|F_{1}\left(P_{k}, x\right)\right|^{2} d x \text { and } \sum_{k} 16 \int_{c\left(P_{k}\right)}\left|F_{2}\left(P_{k}, x\right)\right|^{2} d x .
$$

In fact, for the second sum we will estimate each integral taken over a smaller set than $c\left(P_{k}\right)$. This will be explained after we obtain a bound for the first sum. Notice that the definition of $\mathcal{F}_{1}$ gives that $F_{1}\left(P_{k}, x\right)=f_{1}(x)$ for any $x \in P_{k}$. Then

$$
\sum_{k} 16 \int_{c\left(P_{k}\right)}\left|F_{1}\left(P_{k}, x\right)\right|^{2} d x=\sum_{k} 16 \int_{c\left(P_{k}\right)}\left|f_{1}(x)\right|^{2} d x \leq C \int_{I_{0}}\left|f_{1}(x)\right|^{2} d x .
$$

By almost orthogonality, property (c) for the $\varphi_{(I)}$ 's,

$$
\int_{I_{0}}\left|f_{1}(x)\right|^{2} d x \leq \sum_{J \in \mathcal{F}_{1}} \lambda_{J}^{2}=\int_{I_{0}} \sum_{\substack{J \in \mathcal{F}_{1} \\ J \ni x}} \frac{\lambda_{J}^{2}}{|J|} d x \leq(A \gamma)^{2}\left|I_{0}\right| \leq \frac{\beta}{3}\left|I_{0}\right|
$$

for $\gamma$ sufficiently small. The second to the last estimate follows from the fact that for $x \in I_{0} \backslash \bigcup I_{j}\left(I_{j}\right.$ are the maximal cubes defined at the beginning of the proof), $\sum_{J \ni x} \lambda_{J}^{2} /|J| \leq G^{*}(x)^{2} \leq(A \gamma)^{2}$, and for $x \in I_{j}$, the sum $\sum_{J \ni x, J \in \mathcal{F}_{1}}$ is empty.

Next we bound $\sum_{k}\left|\left\{x \in c\left(P_{k}\right): F_{2}\left(P_{k}, x\right)>0.25\right\}\right|$. As in [W] we cut out a thin annular region around each of the $P_{k}$ 's to handle edge effects. Choosing $\tau>1$ so that $\left|\tau P_{k} \backslash P_{k}\right| \leq(\beta / 3)\left|P_{k}\right|$, and letting $D=\bigcup\left\{\tau P_{k} \backslash P_{k}\right\}$, we have $|D| \leq(\beta / 3)\left|I_{0}\right|$ (remember the $P_{k}$ are disjoint). Also

$$
\sum_{k}\left|\left\{x \in c\left(P_{k}\right): F_{2}\left(P_{k}, x\right)>0.25\right\}\right| \leq|D|+\sum_{k} 16 \int_{c\left(P_{k}\right) \backslash D}\left|F_{2}\left(P_{k}, x\right)\right|^{2} d x .
$$

We need only prove that

$$
\sum_{k} 16 \int_{c\left(P_{k}\right) \backslash D}\left|F_{2}\left(P_{k}, x\right)\right|^{2} d x \leq C^{\prime}(A \gamma)^{2}\left|I_{0}\right|,
$$

and take $\gamma$ small enough so that $C^{\prime}(A \gamma)^{2} \leq \beta / 3$. 
If $k$ is temporarily fixed and $x \in c\left(P_{k}\right) \backslash D$, then $F_{2}\left(P_{k}, x\right)=$ $\sum_{J \in \mathcal{F}_{2}, J \not \subset P_{k}} \lambda_{J} \varphi_{(J)}(x)$, so

$$
\begin{aligned}
\left|F_{2}\left(P_{k}, x\right)\right|^{2} & \leq\left|\sum_{J \in \mathcal{F}_{2}, J \not \subset P_{k}} \lambda_{J} \varphi_{(J)}(x)\right|^{2} \\
& \leq\left(\sum_{J \in \mathcal{F}_{2}, J \not \subset P_{k}}\left|\lambda_{J}\right| l(J)^{2-n / 2}\left(1+\frac{\left|x-x_{J}\right|}{l(J)}\right)^{2-n}\right)^{2}
\end{aligned}
$$

by (a). Again, the Cauchy-Schwarz inequality gives

$$
\begin{aligned}
& \quad \leq\left. F_{2}\left(P_{k}, x\right)\right|^{2} \\
& \left.\leq \sum_{J \subset P_{j}, j \neq k} \frac{\lambda_{J}^{2}}{|J|}\left(1+\frac{\left|x-x_{J}\right|}{l(J)}\right)^{-n}\right) \cdot\left(\sum_{J \subset P_{j}, j \neq k} l(J)^{4}\left(1+\frac{\left|x-x_{J}\right|}{l(J)}\right)^{4-n}\right) \\
& \leq C G\left(P_{k}\right)^{2} \cdot C(\operatorname{diam}(\Omega))^{4} \cdot\left(\sum_{J \subset P_{j}, j \neq k}\left(1+\frac{\left|x-x_{J}\right|}{l(J)}\right)^{-n}\right) .
\end{aligned}
$$

To bound the last sum by a constant we note that $\left|x-x_{J}\right| \geq C\left|x-x_{P_{j}}\right|$ whenever $x \in c\left(P_{k}\right) \backslash D$ and $J \subset P_{j}, j \neq k$. So as above

$$
1+\frac{\left|x-x_{J}\right|}{l(J)} \geq \frac{\left|x-x_{J}\right|}{l(J)} \geq C \frac{\left|x-x_{P_{j}}\right|}{l\left(P_{j}\right)} \cdot \frac{l\left(P_{j}\right)}{l(J)} \geq C^{\prime} \frac{l\left(P_{j}\right)}{l(J)}\left(1+\frac{\left|x-x_{P_{j}}\right|}{l\left(P_{j}\right)}\right),
$$

since also $\left|x-x_{P_{j}}\right| \geq C^{\prime \prime} l\left(P_{j}\right)$. We have

$$
\sum_{J \subset P_{j}, j \neq k}\left(1+\frac{\left|x-x_{J}\right|}{l(J)}\right)^{-n} \leq C \sum_{J \subset P_{j}, j \neq k}\left(\frac{l\left(P_{j}\right)}{l(J)}\right)^{-n}\left(1+\frac{\left|x-x_{P_{j}}\right|}{l\left(P_{j}\right)}\right)^{-n} .
$$

This means that

$$
\begin{aligned}
\sum_{k} \int_{c\left(P_{k}\right) \backslash D}\left|F_{2}\left(P_{k}, x\right)\right|^{2} d x \\
\quad \leq C \sum_{k} \int_{c\left(P_{k}\right) \backslash D} \sum_{j \neq k} \sum_{J \subset P_{j}} \frac{|J|}{\left|P_{j}\right|}\left(1+\frac{\left|x-x_{P_{j}}\right|}{l\left(P_{j}\right)}\right)^{-n} d x \\
\quad \leq C \sum_{I_{0}} \sum_{j}\left(1+\frac{\left|x-x_{P_{j}}\right|}{l\left(P_{j}\right)}\right)^{-n} d x \\
\leq C \sum_{j} \int_{I_{0}}\left(1+\frac{\left|x-x_{P_{j}}\right|}{l\left(P_{j}\right)}\right)^{-n} l\left(P_{j}\right)^{n} d\left(\frac{\left(x-x_{P_{j}}\right)}{l\left(P_{j}\right)}\right) \\
\quad \leq C \sum_{j}\left|P_{j}\right| \int \frac{r^{n-1}}{(1+r)^{n}} d r d \omega_{n-1} \leq C\left|I_{0}\right| \log (1+\operatorname{diam}(\Omega)),
\end{aligned}
$$


using polar coordinates and the fact that the $P_{j}$ 's are disjoint in $I_{0}$. The Central Lemma is proved.

Corollary. Suppose $\sigma \in A^{\infty}\left(Q_{0}, d x\right)$ and $f(x)=\sum_{J \in \mathcal{F}} \lambda_{J} \varphi_{(J)}(x)$ with $\mathcal{F}$ a finite family of cubes from $\mathcal{W}$ and the $\varphi_{(J)}$ satisfying $(\mathrm{a}),\left(\mathrm{a}^{\prime}\right),(\mathrm{b})$ and (c). Then for any $\beta>0$ there exists a $\gamma=\gamma(n, \lambda, \varepsilon, \Omega, \alpha, \beta)$ so that, for every $\xi>0$,

$$
\sigma\left(\left\{x \in Q_{0}: F^{*}(x)>2 \xi, G^{*}(x) \leq \gamma \xi\right\}\right) \leq \beta \sigma\left(\left\{x \in Q_{0}: F^{*}(x)>\xi\right\}\right) .
$$

Proof. Let $\left\{I_{j}\right\}$ be the maximal dyadic cubes in $Q_{0}$ such that $F\left(I_{j}\right)>\xi$. We need only show that

$$
\left|\left\{x \in I_{j}: F^{*}(x)>2 \xi, G^{*}(x) \leq \gamma \xi\right\}\right| \leq \widehat{\beta}\left|\left\{x \in I_{j}: F^{*}(x)>\xi\right\}\right|
$$

for some $\widehat{\beta}$ such that $\left(C_{0} \widehat{\beta}\right)^{1 / \kappa} \leq \beta$, because $\sigma \in A^{\infty}(d x)$. Notice that $\left\{x \in Q_{0}: F^{*}(x)>\xi\right\}=\bigcup I_{j}$. Once again we cut out a small annular region for each cube $I_{j}$, but here the region lies inside $I_{j}$. We take $\varepsilon>0$ so small that $\left|\left\{x \in I_{j}: \operatorname{dist}\left(x, I_{j}^{\mathrm{c}}\right) \leq \varepsilon\right\}\right| \leq(\widehat{\beta} / 3)\left|I_{j}\right|$. For $x \in(1-\varepsilon) I_{j}$ we have $\left|F\left(I_{j}\right)-F\left(I_{j}, x\right)\right| \leq C G\left(I_{j}\right)$, by Lemma 4 . It is also true that for $\widehat{I_{j}} \supset I_{j}$ with $l\left(I_{j}\right)=0.5 l\left(\widehat{I_{j}}\right)$, Lemma 7 implies that $\left|F\left(\widehat{I}_{j}\right)-F\left(I_{j}\right)\right| \leq C^{\prime} G\left(I_{j}\right)$. By maximality $F\left(\widehat{I_{j}}\right) \leq \xi$. We also have

$$
\begin{aligned}
E_{j}= & \left\{x \in I_{j}: F^{*}(x)>2 \xi, G^{*}(x) \leq \gamma \xi\right\} \\
\subset & \left\{x \in(1-\varepsilon) I_{j}: F^{*}(x)>2 \xi, G^{*}(x) \leq \gamma \xi\right\} \\
& \cup\left\{x \in I_{j}: \operatorname{dist}\left(x, I_{j}^{\mathrm{c}}\right) \leq \varepsilon\right\} .
\end{aligned}
$$

For any $I_{j}$ such that $E_{j} \neq \emptyset$, we have $G\left(I_{j}\right) \leq \gamma \xi$. From the previous calculations we obtain $\left|F\left(I_{j}, x\right)\right| \leq F\left(\widehat{I_{j}}\right)+c G\left(I_{j}\right)$ for any $x \in \eta I_{j}$. So if $\gamma$ is small enough then $\left|F\left(I_{j}, x\right)\right| \leq 1.2 \xi$. Writing

$$
f(x)=\sum_{J \not \subset I_{j}, J \in \mathcal{F}} \lambda_{J} \varphi_{(J)}(x)+\sum_{J \subset I_{j}, J \in \mathcal{F}} \lambda_{J} \varphi_{(J)}(x)=F\left(I_{j}, x\right)+h(x),
$$

we get

$$
F^{*}(x)-1.2 \xi \leq H^{*}(x) \quad \text { with } \quad H^{*}(x)=\sup _{I \ni x} H\left(I, x_{I}\right) .
$$

This happens since $I_{j}$ is maximal so that $F\left(I_{j}\right)>\xi$; consequently, any dyadic cube $Q \ni x$ such that $F(Q)>2 \xi$ must be contained in the $I_{j}$ that contains $x$. Setting $F_{j}(x)=F\left(I_{j}, x\right)$, we have

$$
\sup _{\substack{J \ni x \\ J \subset I_{j}}} F_{j}\left(J, x_{J}\right)=\sup _{\substack{J \ni x \\ J \subset I_{j}}} F_{j}\left(x_{J}\right) .
$$

Also, $x \in(1-\varepsilon) I_{j}$ means that for any dyadic $J \subset I_{j}$ such that $x \in J$, $\operatorname{dist}\left(x_{J}, I_{j}^{\mathrm{c}}\right) \geq(\varepsilon / 2) l\left(I_{j}\right)$. Taking $\eta=1-\varepsilon / 2$, we have $F_{j}\left(x_{J}\right) \leq 1.2 \xi$ for any 
such $J$, so

$$
\begin{aligned}
\left\{x \in(1-\varepsilon) I_{j}: F^{*}(x)\right. & \left.>2 \xi, G^{*}(x) \leq \gamma \xi\right\} \\
& \subset\left\{x \in(1-\varepsilon) I_{j}: H^{*}(x)>0.8 \xi, G^{*}(x) \leq \gamma \xi\right\} .
\end{aligned}
$$

After rescaling, the Central Lemma can be applied to the function $h(x)$.

The full result of Theorem 1 follows from the Corollary by a standard argument because $\Omega$ is bounded and $f(x)$ being a finite sum, means that $F^{*} \in L^{p}(\Omega, d \sigma)$. To prove Theorem 1 for infinite sums we can use Fatou's lemma on $\left|f_{n}(x)\right|^{p}$, for $f_{n}(x)=\sum_{J \in \mathcal{F}, l(J) \geq 1 / n} \lambda_{J} \varphi_{(J)}(x)$, taking $\mathcal{F}$ to be an infinite family of dyadic cubes from $\mathcal{W}$.

\section{References}

[A] A. A. Arkhipova, Reverse Hölder inequalities with boundary integrals and $L_{p}$ estimates for solutions of nonlinear elliptic and parabolic boundary value problems, in: Nonlinear Evolution Equations, Amer. Math. Soc. Transl. 164, Amer. Math. Soc., 1995, 15-42.

[CF] R. Coifman and C. Fefferman, Weighted norm inequalities for maximal functions and singular integrals, Studia Math. 51 (1974), 241-250.

[GM] M. Giaquinta and M. Modica, Regularity results for some classes of higher order nonlinear elliptic systems, J. Reine Angew. Math. 311/312 (1979), 145-169.

[GW] M. Grüter and K-O. Widman, The Green function for uniformly elliptic equations, Manuscripta Math. 37 (1982), 303-342.

[K] C. Kenig, Harmonic Analysis Techniques for Second Order Elliptic Boundary Value Problems, Amer. Math. Soc., Providence, RI, 1994.

[S] C. Sweezy, Weights and Hölder norms for solutions to a second order elliptic Dirichlet problem on nonsmooth domains, in: Proc. Prairie Analysis Seminar 2005, Amer. Math. Soc., to appear.

[SW] C. Sweezy and J. M. Wilson, Weighted norm inequalities for parabolic gradients on nonsmooth domains, Int. J. Pure Appl. Math. 24 (2005), 61-109.

[WW] R. L. Wheeden and J. M. Wilson, Weighted norm estimates for gradients of half-space extensions, Indiana Univ. Math. J. 44 (1995), 917-969.

[W] J. M. Wilson, Global orthogonality implies local almost orthogonality, Rev. Math. Iberoamer. 16 (2000), 29-48.

Department of Mathematical Sciences

New Mexico State University

Box $300013 \mathrm{MB}$

Las Cruces, NM 88003-8001, U.S.A.

E-mail: csweezy@nmsu.edu 